\title{
A Novel Framework for Highlight Reflectance Transformation Imaging
}

\author{
Andrea Giachetti $^{\mathrm{a}}$, Irina Ciortan ${ }^{\mathrm{a}}$, Claudia Daffara $^{\mathrm{a}}$, Giacomo Marchioro ${ }^{\mathrm{a}}$, Ruggero Pintus ${ }^{\mathrm{b}}$, Enrico Gobbetti $^{\mathrm{b}}$ \\ ${ }^{a}$ University of Verona, Italy \\ http://www.univrit/ \\ ${ }^{b}$ CRS4, Visual Computing Group, Italy \\ http://www.crs4.it/vic/
}

\begin{abstract}
We propose a novel pipeline and related software tools for processing the multi-light image collections (MLICs) acquired in different application contexts to obtain shape and appearance information of captured surfaces, as well as to derive compact relightable representations of them. Our pipeline extends the popular Highlight Reflectance Transformation Imaging (H-RTI) framework, which is widely used in the Cultural Heritage domain. We support, in particular, perspective camera modeling, per-pixel interpolated light direction estimation, as well as light normalization correcting vignetting and uneven non-directional illumination. Furthermore, we propose two novel easy-to-use software tools to simplify all processing steps. The tools, in addition to support easy processing and encoding of pixel data, implement a variety of visualizations, as well as multiple reflectance-model-fitting options. Experimental tests on synthetic and real-world MLICs demonstrate the usefulness of the novel algorithmic framework and the potential benefits of the proposed tools for end-user applications.
\end{abstract}

Keywords: Multi Light Image Collections, Highlight Reflectance Transformation Imaging, Photometric Stereo, Image Enhancement

\section{Introduction}

Multi-light image collections (MLICs) are an effective mean to gather detailed information on the shape and appearance of objects. They are, thus, widely used in many application contexts.

The basic idea of the approach is to visually characterize objects by capturing multiple images of the surface of interest from a fixed point of view, changing the illumination conditions at each shot. The acquired data is then processed to extract shape and material information. While some techniques exist for general variable environmental illumination [1, 2], the most widespread approach in most application fields, including Cultural Heritage $(\mathrm{CH})$ [3], medical interventions $[4,5]$, and underwater data gathering [6], considers a single calibrated camera taking multiple images of a scene illuminated by a single moving light. A large variety of efficient computational tools have been devised in this context to extract information from the captured image stack in order to effectively solve different problems, such as feature detection and enhancement, reconstruction of normals and 3D shapes, and creation of relightable images.

Photometric Stereo (PS) is probably the most widely known technology based on MLICs. It exploits priors on reflectance functions to derive local normals and global 3D shapes [7]. Reflectance Transformation Imaging (RTI) [8, $9,10,11]$ extends the PS idea by interpolating MLIC reflectance data with parametric functions (Polynomial Texture Maps, PTM; Hemispherical Harmonics, HSH; Discrete Modal Decomposition, DMD), which can be used to estimate and display images relighted from arbitrary angles and incorporating other image enhancements for visually revealing surface details not detectable from a single view [10,12,13]. Other techniques try, rather, to improve understanding by highlighting specific making surface and material properties. For instance, Raskar et al. [14] exploited multilight images to extract depth edges with a simple heuristics and used the result to create non-photorealistic rendering methods, while Fattal et al. [15] used them to generate enhanced images emphasizing shape and surface detail.

RTI is possibly the most widely applied MLIC technique. This kind of imaging has rapidly become a widely used solution for the documentation, recording and decoding of Cultural Heritage $(\mathrm{CH})$ objects, as it supports an offline analysis of the artifacts, supporting and going beyond simulated raking light analysis, and allows the estimation of 
image enhancements emphasizing details $[16,17]$. Furthermore, the reflectance interpolation coefficients derived from MLIC processing, or the image features extracted from the image stack, can be used to characterize and classify materials, as shown in a number of works $[18,19,20]$.

The widespread use of RTI for visual surface characterization, especially in the CH domain, is also due to the fact that it can be performed with a low-cost, flexible, and easy to use setup based on freehand light positioning and highlight-based light direction estimation (H-RTI) [3]. In the H-RTI image-capture technique, the reflection of the light source on one or more reflective spheres visible in each shot enables the processing software to calculate the light direction for each image, providing great robustness and flexibility in subject size and location.

The classic H-RTI acquisition setup and processing pipeline, however, are based on strong assumptions on lights (ideally constant in direction and intensity) and camera model (orthographic), not necessarily matching typical acquisition conditions [3, 21]. In particular, due to the lack of uniformity in illumination intensity and direction, the results obtained with this simple setup may vary widely between acquisitions, and may be unsuitable for quantitative analyses, which include normal estimation, roughness or material segmentation/classification, as well as monitoring over time. Exploitation of H-RTI data is thus often limited to rough qualitative analysis of single acquisitions.

In this article, we revise the H-RTI approach, presenting a novel practical setup and a set of tools that relax the aforementioned strong assumptions. Our solution offers a better support for qualitative analysis of MLICs and enables the addition of quantitative analysis on top of the classic RTI method. Our main contributions are the following:

- a novel practical setup and processing pipeline that can cope with the effects of perspective camera distortion, non-point lights, spatially varying illumination, variable light distance, as well as camera vignetting. Per-pixel light directions are estimated from highlights on multiple reflective spheres, taking into account perspective correction and performing direction interpolation, while illumination variations are compensated by an algorithm exploiting light intensity measured on matte white targets positioned around the object of interest.

- An easy to use tool to perform/control all the processing pipeline, not requiring to rely on external image processing applications and storing reordered pixel information with associated light directions in a dedicated structure that can be effectively used for post-processing (e.g. Photometric Stereo, RTI, feature detection, and visualization).

- An easy to use tool to complete the pipeline with enhanced visualizations, as well as with shape and material information recovery operations.

Our novel combination of several flexible auto-calibration techniques into a single framework aims to provide a significant step towards a practical quantitative and repeatable analysis using simple and low-cost free-form acquisition strategies.

The paper is organized as follows. Sec. 2 provides a quick overview of existing RTI acquisition setups and light calibration approaches, while in Sec. 3 our algorithmic contribution is described. The tools that implement the proposed approach are presented in Sec. 4 . Sec. 5 demonstrates, with experimental tests, the advantages of our improved pipeline, as well as the potential advantages of its use for practical applications.

\section{Related work}

Multi-light acquisition, processing, and analysis are broad research subjects, and a full review is out-of-scope for this article. We concentrate here only on the most-related methods to perform RTI acquisition and processing. For a wider coverage, we refer the reader to established surveys in surface reflectance capture [22], multi-light computational frameworks [7], digital modeling of material appearance [23], and geometric analysis in cultural heritage [24].

A wide variety of RTI acquisition setups exist, ranging from low-cost and transportable kits [21] to different sizes of fixed light domes[21, 25, 26]. Recently, some dome solutions have been presented that use both visible and invisible light wavelengths [27, 28]. Dome solutions allow for pre-calibration of lights, but they are, in general, expensive and not flexible, thus limiting the potential changes in light numbers, positions and types, and the size of captured surfaces.

Our goal is, rather, to improve the classic hand-held light capture, which is low-cost, simple to implement, and allows for a more flexible choice of the number and the positions of the light sources; these factors are very important, for example, when dealing with non-Lambertian, shiny materials. Moreover, it is easy to extend the presented pipeline 
to the multi- or hyper-spectral domain at a much lower cost than multiple-light setups, which can easily require hundreds of illuminators or filters.

Free-form hand-held setups are widely used in the Cultural Heritage domain as powerful Computational Photography tools by many end users, especially to create relightable images and for detail enhancement. This large diffusion is mainly due to publicly available packages such as RTIBuilder and RTIViewer [21], which employ the H-RTI capture setup and use manual annotation of reflective spheres and highlight-based light direction estimation. However, these tools rely on limiting assumptions about lighting and camera, i.e., uniform and far point light (collimated rays) and orthographic camera with an ideal lens. Since the computation of surface attributes leads to significant errors and provides variable results for each acquisition, the applications of this method for geometrical reconstruction, material acquisition, and quantitative analysis are limited. Conversely, we want to adopt here more realistic lighting and camera models, taking into account optical effects such as vignetting, non-uniform light emission, and light attenuation with distance.

The calibration of real illumination is a well-known topic in Computer Vision, and, specifically, in the Photometric Stereo (PS) field [7]. While some methods try to implicitly consider real light sources within the particular PS framework [29, 30], others are more focused on the explicit calibration of various lighting properties. Some methods make assumptions on light form factor, e.g., near point light [31] or linear light source [32], and try to exploit the illuminated scene to extract the light position and direction. For instance, Ahmad et al. [31] exploit diffused maxima regions in the framed model, and derive from them the light directions. Others perform calibration by sampling light on calibration targets of known shape and albedo (e.g., reflective spheres or diffuse targets). Corsini et al. [33] use high-dynamic range images of two reflective balls to acquire the spatially-varying illumination of a real-world scene, and it focuses more on the environment light effect rather than of the computation of a per-pixel light direction and intensity. Ackermann et al. [34] present a study and validation through error statistics of both a forward and backward geometric point light source calibration by using sets of different numbers of reflective spheres. Although it proposes a very simple and robust way to compute light direction, it considers a point light model without taking into account non-uniform light intensity. Other methods strongly rely on a specific, fixed light form factor (e.g., LED light [35, 36], and model the intensity with the corresponding fall-off due to both distance and angle to the light principal axis. Xie et al. [36] also consider vignetting effects. Unfortunately, those methods are not applicable to the case of a general variable illumination due to non-ideal lamps or lenses. Some works thus try to cope with non-uniform intensity without imposing an analytical light model $[37,38]$. Similarly to us, they use a flat reference object with known albedo to sample an arbitrary lighting vector field and to calibrate it using a flat-fielding approach. They don't use polynomial interpolation, but they exploit measured spatially-varying intensities to compensate the input images, and to convert the problem into a standard collimated case. Differently to our work, they require different acquisitions for the calibration step and the actual capture; this is possible only with a fixed light configuration, but it is not applicable to a more general free-form, hand-held multi-light acquisition. In our approach, we use multiple spheres to estimate a light direction field, and use measures on a planar white target to estimate the intensity of each light ray, infilling missing data with a low-degree interpolation, thus reconstructing an approximation of the entire light field illuminating the scene.

The pipeline presented here was preliminarily proposed in our previous conference papers [39, 20]. The pipeline improves the classical highlight-based RTI capture framework by estimating per-pixel interpolated light direction and creating intensity-corrected images simulating constant illumination on a reference plane. We here provide a more thorough exposition, but also significant new material, including the support for a non-orthographic camera model, a new organization of data that facilitates processing and display, the presentation of easy-to-use software interfaces to perform all the processing steps and novel experiments to demonstrate the advantages of the proposed methods. Finally, we have attempted to further clarify the steps in our methods to facilitate their implementation and to make the transfer between abstract concepts and actual code as straightforward as possible.

\section{Improved Highlight RTI pipeline}

Our complete acquisition and processing pipeline is shown in Fig. 1. We acquire and take as input a MultiLight image collection. Light information may be in principle known for each image if coming from a calibrated system (light dome). If lights are not known and calibrated, as in hand-held light acquisition, the classical solution 


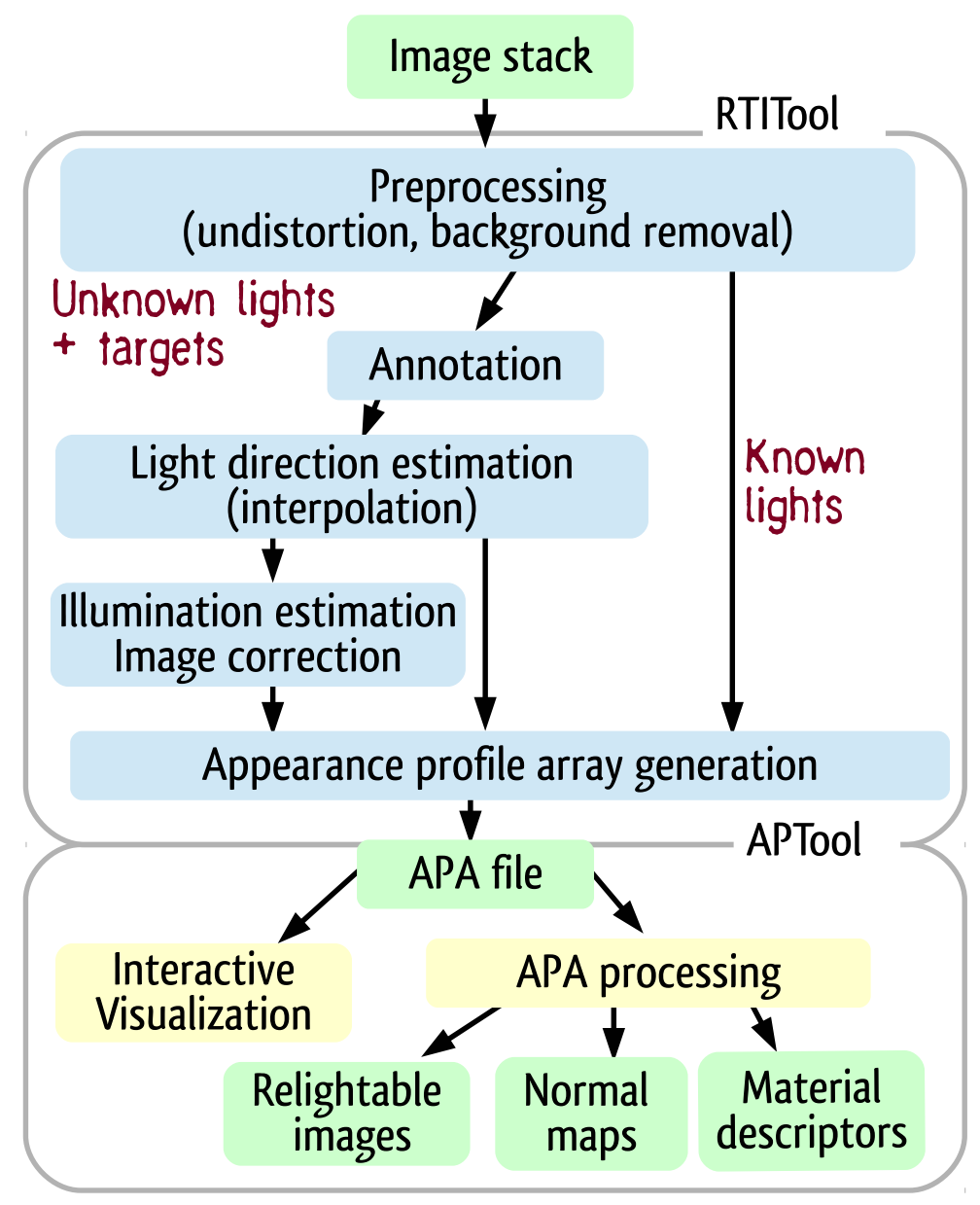

Figure 1: The proposed MLIC processing pipeline.

is to assume uniform intensity and direction and use a reflective sphere for estimating light direction from highlight position (H-RTI).

Our first contribution is a more complete setup (Fig. 2(a)) to characterize lights directly from images, improving H-RTI. This setup includes, in addition to several (typically four) reflective spheres, a matte white planar frame around the object being captured. The multiple spheres are used to derive a more accurate per pixel interpolated direction, while the frame is used to estimate a correction for light non-uniformity and vignetting, as described in Sec. 3.4. Several instantiations of this concept are possible. In particular, if an object is captured in a typical laboratory setup, the white frame can be replaced by a Lambertian surface covering the plane supporting the object. Moreover, in outdoor acquisitions of large objects, spheres at the corner of the visual fields and multiple co-planar Lambertian targets on the acquisition reference plane could be placed, as well, and used for the subsequent calibration procedures. In order to simplify generic on-site acquisitions, we realized a modular frame building set, which combines 3D-printed supports for spheres with aluminum bars of different lengths covered by approximately Lambertian coating (Fig. 2(b)). This allows the creation of rigid frames that can be placed in horizontal, vertical or arbitrary orientations. The current version holds $5 \mathrm{~cm}$ wide spheres, but we plan to realize sets of different sizes.

Before the acquisition, we assume that we have already (and once) calibrated the internal characteristics of the camera, in order to obtain the radiometric response function and the lens parameters. The capture process outputs an image stack, which is preprocessed and semi-automatically annotated with custom software (see Sec. 4) to find the position of the spheres and rectangular regions of the white frame (Fig. 3). From the positions of highlights, the incident light direction is estimated at the highlight location and interpolated across the whole image. Then, 


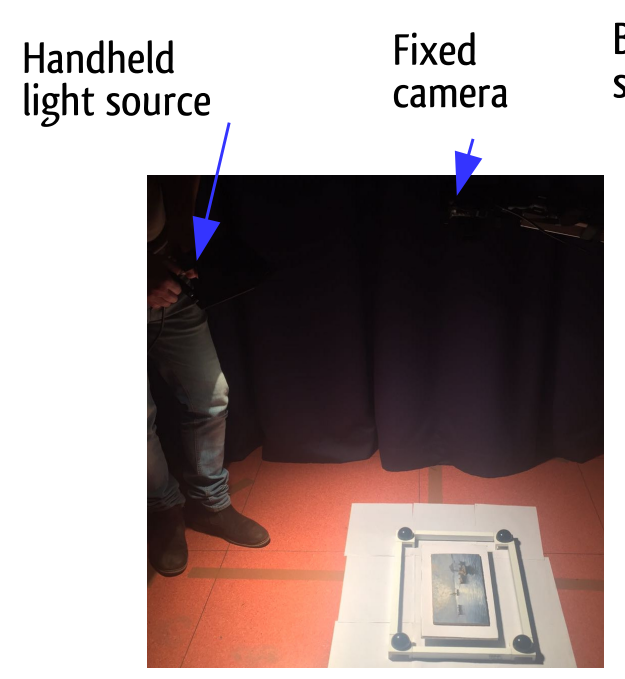

(a) Setup

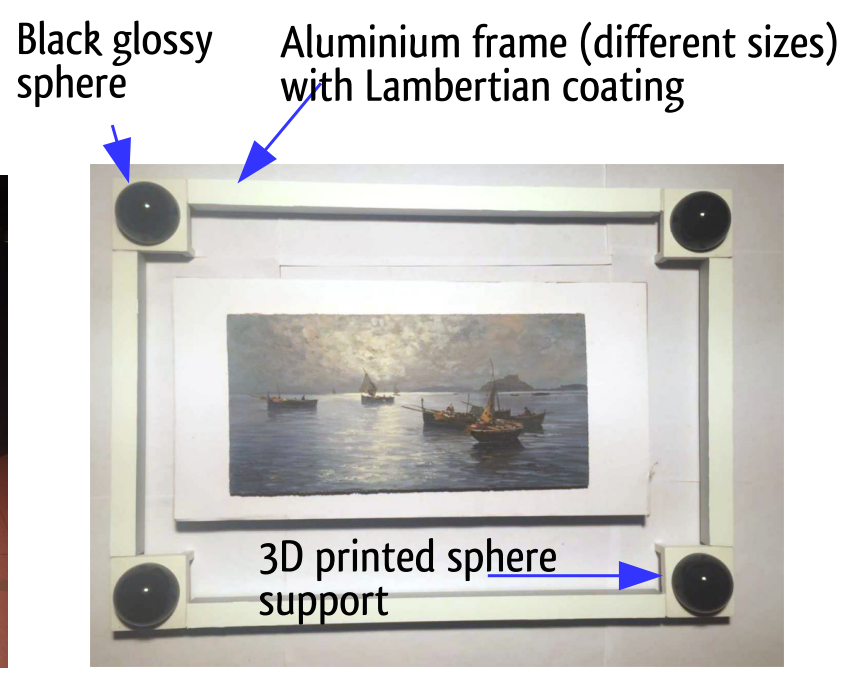

(b) Scene

Figure 2: Capture setup. (a) The acquisition setup consists in a stationary DSLR digital camera, a hand-held light source, some calibration targets (a Lambertian white background/frame and four black reflective spheres), and the object being acquired positioned on a planar support perpendicular to the camera axis. (b) Camera view of the scene.

illumination intensity is corrected at each pixel location. This is done by multiplying the local value by the factor that would make the locally interpolated white frame intensity match a reference value multiplied by the cosine of the local light direction elevation angle.

After that, each pixel is associated with a calibrated reflectance profile (appearance profile), coupled with calibrated light parameters. Those are used to provide the user with an interactive data visualization, and to perform various processing operations on reflectance data. For instance, as in typical RTI settings, we fit reflectance data to a low-dimensional analytic representation, in order to extract a small set of coefficients that can compactly describe the image stack at each pixel. Then, we use this information to relight the object, to compute geometric attributes (e.g., normal maps or 3D surface reconstruction), or to extract meaningful appearance features and descriptors for material classification and recognition.

All the procedures can be controlled by two software tools that will be described in detail in Sec. 4: one dedicated to the preprocessing and reorganization of pixel data (RTITool), one to reflectance data fitting, normals estimation, visualization and analysis (APTool).

In the rest of this section, we provide details on the major pipeline components: preprocessing to prepare data for further elaboration (Sec. 3.1), perspective light direction estimation from highlight on a single sphere (Sec. 3.2), reconstruction of lper-pixel light direction by interpolation of results on multiple detected highlights (Sec. 3.3), light intensity correction by exploiting interpolated directions and measures on a matte planar target (Sec. 3.4), storage of the calibrated per-pixel information in a 3D appearance profile array (Sec. 3.5), and, finally, basic processing of appearance profile data to recover shape and reflectance parameters (Sec. 3.6).

\subsection{Preprocessing}

Image preprocessing consists mainly in the removal of ambient light and undistortion. These two transformations are applied to all the images in the collection before they are fed to the light direction estimation step. The ambient light is captured by acquiring an extra image of the scene with the handheld light source turned off. The undistortion is performed according to the intrinsic camera parameters estimated a priori with a standard calibration procedure. Auxiliary to the annotation of the black reflective spheres and the white Lambertian frame, stand the maximum image and respectively, the minimum image estimation. The maximum of the image collection discards the shadow around the spheres, hence improving the visual acuity, while the minimum image maps the projected shadows areas that should be avoided when selecting consistent highly reflective regions (Fig. 3). 


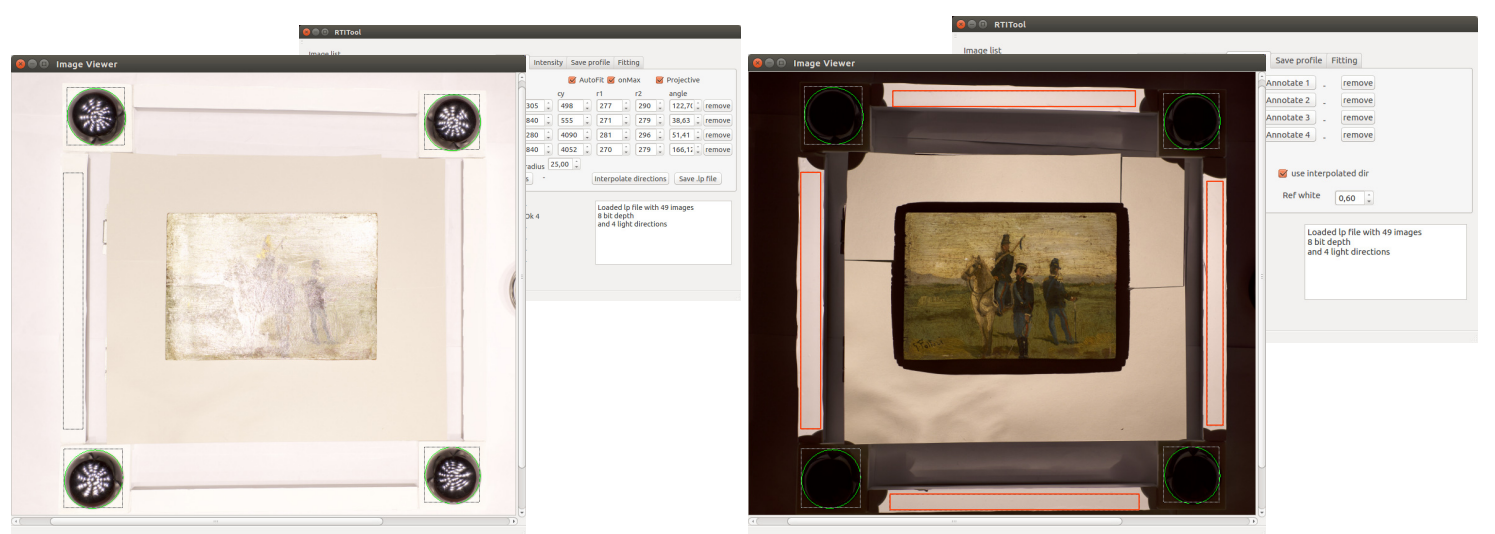

Figure 3: Snapshots of the RTITool interface during the annotation of reflective spheres (left) over the maximum image estimated from the MLIC stack, and the annotation of the Lambertian frame performed on the minimum image estimates from the stack (right).

\subsection{Perspective Light direction estimation}

In the general case of free-form RTI acquisition without known lights, we compute the highlight-based light direction by releasing the orthographic projection hypothesis used in previous classic solutions [3] and implemented in the well-known RTIBuilder package. This allows the computation of light direction when the reflective spheres are at the margin of the image and appear relevantly distorted (elliptical) in the image.

In the current algorithm and implementation, we assume known intrinsic parameters of the camera: optical center, $\overrightarrow{o_{x}}, \overrightarrow{o_{y}}$, pixel size, $\overrightarrow{s_{x}}, \overrightarrow{s_{y}}$, and focal length $\vec{f}$. They are loaded from files in the software tool. However, if we have a scene with multiple reflective spheres, we could, in principle, exploit them also to calibrate the camera including distortion parameters [40]. We plan to include this feature in future version of the package.

Once we have identified the projected sphere outline, that is an ellipse, we can easily locate the extrema of the major axes, with known coordinates in camera frame $\vec{p}=\left(\left(p_{x}-o_{x}\right) s_{x},\left(p_{y}-o_{y}\right) s_{y}, f\right)$ and $\vec{q}$ (Fig. 4). Note that the knowledge of the pixel size $\overrightarrow{s_{x}}, \overrightarrow{s_{y}}$ is not necessary. We can only add knowledge of aspect ratio $s$ to the focal length expressed in pixels. From $\vec{p}$ and $\vec{q}$, we can easily compute the direction of the vectors $\vec{a}, \vec{b}$ pointing to the corresponding tangent points on the sphere $\vec{P}, \vec{Q}$

$$
\begin{aligned}
& \vec{a}=(\vec{p}-\vec{O}) /\|\vec{p}-\vec{O}\| \\
& \vec{b}=(\vec{q}-\vec{O}) /\|\vec{q}-\vec{O}\|
\end{aligned}
$$

This also allows us to estimate the unit vector $\vec{w}$ pointing to the center of the sphere $\vec{C}$ :

$$
\vec{w}=(\vec{a}+\vec{b}) /\|\vec{a}+\vec{b}\|
$$

The triangle $\overrightarrow{O C} \vec{P}$ has known angles $\arcsin (\vec{a} \cdot \vec{w}), \arccos (\vec{a} \cdot \vec{w}), \pi / 2)$ and a known side $R$. We can, thus, estimate the distance $D$ of the sphere center from the camera center, and the coordinates of the sphere center in camera coordinates $C=D \vec{w}$.

Since we have multiple spheres on a plane, we can then estimate the plane orientation/position from the estimated centers with a simple fit.

Once we estimate the position of the projected highlight $\vec{h}$, we can solve for the $3 \mathrm{D}$ highlight position and the light direction estimation, by computing the view unit vector

$$
\vec{v}=(\vec{o}-\vec{h}) /\|\vec{o}-\vec{h}\|
$$

and the equation of the line from the origin to the highlight

$$
\vec{X}=-t \vec{v}
$$




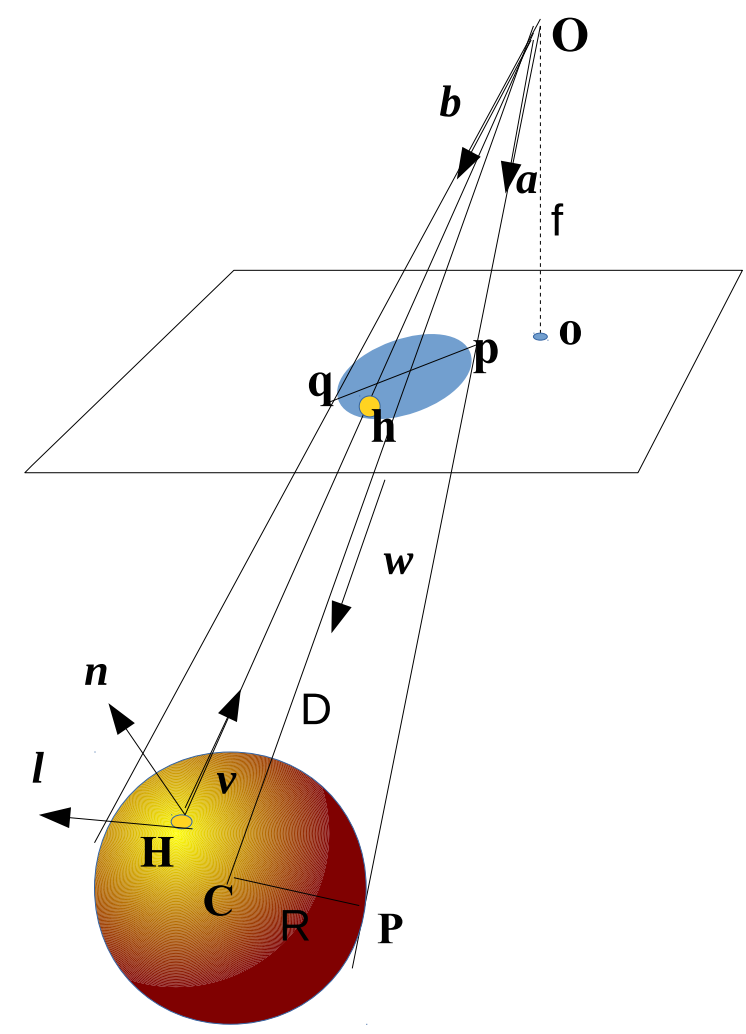

Figure 4: Light direction can be easily computed also assuming releasing the orthographic projection constraint.

Solving the equation system that combines this equation and the sphere equation

$$
\left(X_{1}-C_{1}\right)^{2}+\left(X_{2}-C_{2}\right)^{2}+\left(X_{3}-C_{3}\right)^{2}=R^{2}
$$

we can find two intersections. The one closest to the origin is the highlight position in $3 \mathrm{D} \vec{H}$. The unit normal in the point is then

$$
\vec{n}=(\vec{H}-\vec{C}) /\|\vec{H}-\vec{C}\|
$$

and reflecting $\vec{v}$ with respect to $\vec{n}$ we can estimate the light direction $\vec{l}$.

In our tool, we implemented this algorithm coupled with a simple ellipse detector based on local image binarization and ellipse fitting obtained with OpenCV implementation of Fitzgibbon's method[41].

\subsection{Multiple spheres setup and light direction interpolation}

By putting a sphere at the margin of the image, we reduce the odds that it casts shadow on the object. The perspective model allows us to do this even in non-ideal conditions and wide field of views.

For most light sources, in addition, the assumptions of a parallel and uniform beam across the entire scene is also far from being fulfilled, and errors introduced in this case are not negligible, as shown in the experimental section. $\mathrm{We}$, therefore, strive to obtain, when possible, a better per-pixel light direction estimation by using multiple (typically four) spheres placed close to image corners, estimating directions for the various highlight positions, and linearly interpolating estimated light direction across the image.

If this configuration is chosen, rather than just estimate and store a light direction for each image, we estimate for each image the coefficients of a linear interpolation of the directions that are later used to recover per pixel light direction values. Coefficients are saved in our specialized data structure (appearance profile array, APA) and used to support a better estimation of reflectance parameters. 


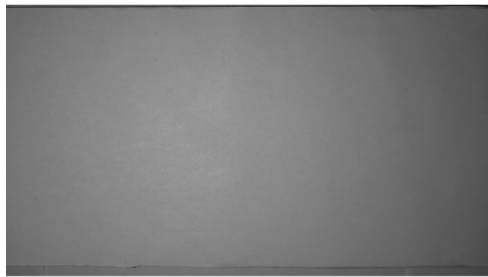

(a)

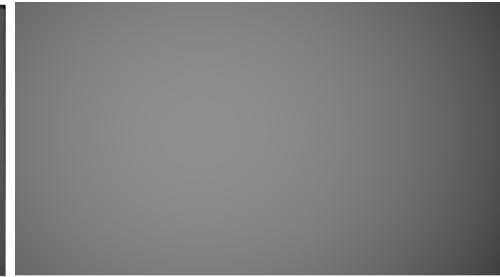

(b)

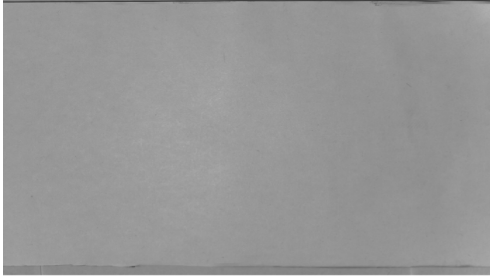

(c)

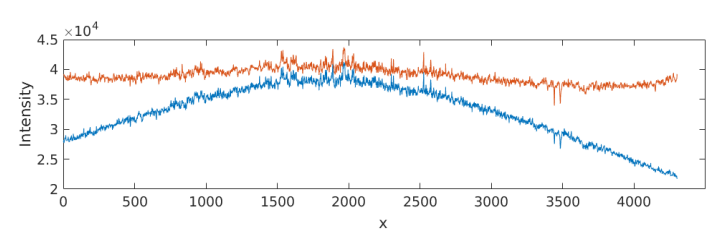

(d)

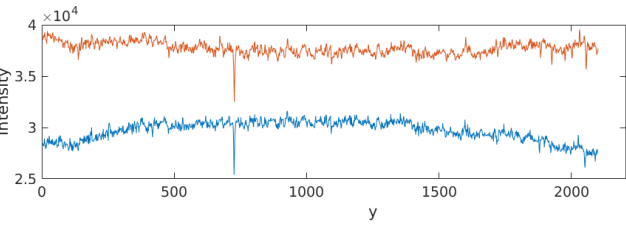

(e)

Figure 5: Intensity correction procedure of image with only white background: from the annotated planar frame (not visible in the cropped image) at the border of the original image (a), a polynomial estimate of the illumination in the whole image is performed (b), and used to estimate a corrected image (c). Intensity profiles along the central line and column in the original and corrected images are compared in (d),(e).

\subsection{Light intensity correction}

The non-uniformity of the beam intensity can be reasonably corrected with a solution that can be applied in many practical acquisition scenarios. The idea, here, is to place a planar frame around the object of interest, with an approximately Lambertian coating. By detecting the region in the images where the target is illuminated, excluding the parts that can be shadowed in some images, we can use the measured pixel values on the target to calibrate the pixel values on the acquired objects, in order to simulate an acquisition made with a truly constant light intensity, at least on the plane of the frame. Ideally, for a Lambertian surface, the brightness of the region should be constant (if the light direction is constant). In practice, we measure a non-negligible non-uniformity using common lights and cameras, due to non-uniformity of the light beam, as well as to vignetting effects of the lenses.

By fitting a polynomial function of the light direction over the brightness estimated in the frame regions, we can compute a correction factor for the estimated light, making the image illuminated as if the light intensity has a standard reference value, and the local light direction estimated at each pixel location (we obtain this by weighting the expected Lambertian reflectance of paper by the actual local cosine factor). This light normalization can correct different non-uniformity causes. Of course it assumes that the light intensity is not changing with depth in the region of interest. Since beam variations are expected to be smooth, we use a quadratic interpolation of the reflectance to extend the reference illumination to the entire plane of interest starting from the reference values on the target. It should be noted that even if the current software fits a quadratic model, a more complex function will be investigated in the future. Fig. 5 shows the effect of the correction procedure in an image with only a planar diffusive surface (spheres and calibration frames were outside the cropped region of interest). The procedure successfully flattens the intensity profiles due to spotlight shape and vignetting.

\subsection{Appearance profile array files storage}

In order to simplify data processing steps, we store the data stack after in a reorganized array structure, where all the per-pixel information is represented sequentially to allow model fitting or pixel processing without the necessity of loading all the data in memory or to allocate large array in processing software. The file structure used (appearance profile array, APA) is composed of a header and data section. The header describes the encoding choices (8 or 16 bits, RGB or chromaticity+luminance, constant or interpolated light directions) and the light direction information (vector elements or interpolation coefficients). The data section stores pixel values in a 3D array. Fig. 6 shows the information encoded in appearance profile: all the brightness information of a pixel location is stored together and can be represented in $l_{x}, l_{y}$ coordinates and interpolated for a better display. The shape of the resulting function is characteristic of both shape and material properties. We tested both Delaunay Triangulation based interpolation and 


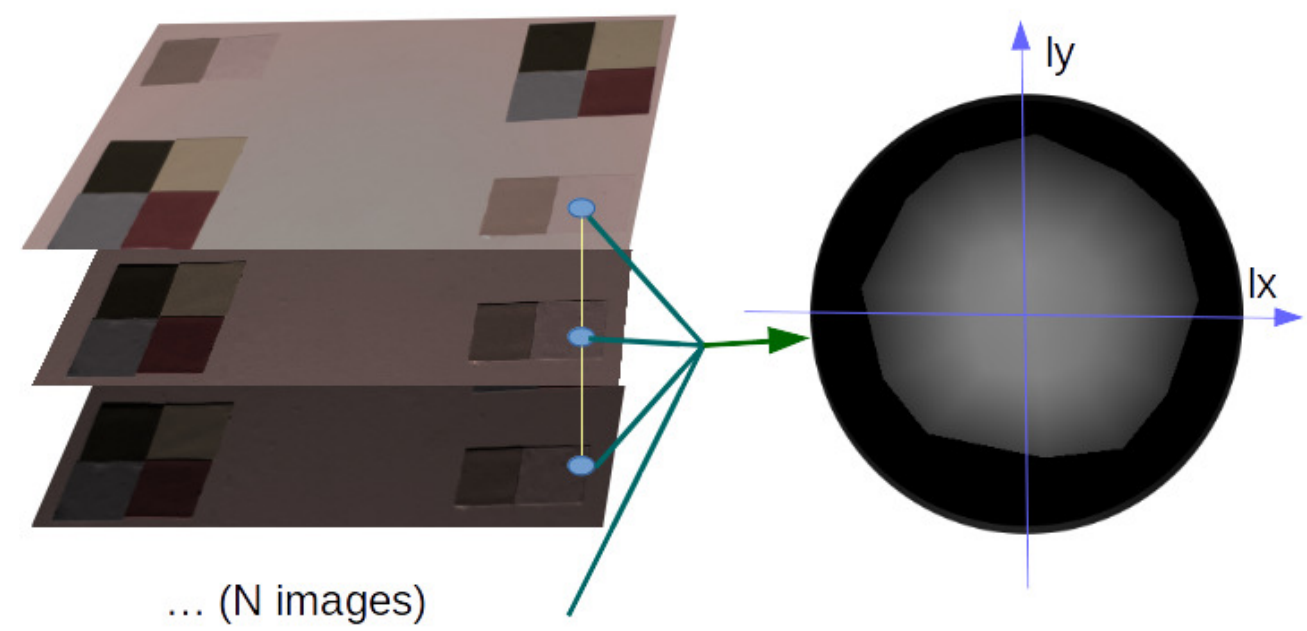

Figure 6: Appearance profile visualization using Delaunay Triangulation based interpolation of the intensity values at the pixel location in the $l_{x}, l_{y}$ plane.

Radial basis functions to obtain visualizations of the local appearance map. Using these interpolation algorithms, relighted images can thus also be directly displayed without the need for simplified parametric representations of the local reflectance as a function of light direction.

\subsection{MLIC data basic processing: Photometric stereo, PTM/HSH fitting}

Apart from creating relighted images with interpolation in the light direction space, the MLIC stacks encoded as (intensity-corrected) appearance profiles can be processed with the standard algorithms used to recover shape and reflectance parameters. Given for each pixel the light direction $\left(l_{x}(i), l_{y}(i), l_{z}(i)\right)$ and the (corrected) reflectance $L(i)$ known for $N$ light directions $\vec{l}(i)$, basic Photometric Stereo estimates albedo $a$ and normals $\vec{n}$ assuming the Lambertian model and solving the overconstrained system

$$
L(i)=a\left[n_{x}, n_{y}, n_{z}\right]\left[l_{x}(i), l_{y}(i), l_{z}(i)\right]^{T} i=1 \ldots N
$$

PTM fitting approximates the reflectance function with a polynomial function, also using a least squares solution to find coefficients. The classical form [10] is

$$
L(i)=[a, b, c, d, e, f]\left[l_{x}^{2}(i), l_{y}^{2}(i), l_{x}(i) l_{y}(i), l_{x}(i), l_{y}(i), 1\right]^{T}
$$

but different polynomial function have been proposed, as well as different fitting functions, such as Hemispherical Harmonics or Discrete Modal Decomposition [12, 13]. Implementing different function fitting is quite simple, and their ability to represent the real reflective behavior of the material depends clearly on the kind of material analyzed.

Furthermore, it must be considered that non-local effects, such as interreflections and projected shadows, create local anomalous behaviors of the laws directly linking light angle and reflected color. To cope with these effects, and also to separate diffusive behavior from specular highlights, robust fitting methods have been proposed [42, 43], trying to remove outliers from the parameters estimation procedure.

\section{Simple tools for RTI data processing}

We designed two software tools to process image stacks captured by a camera in RTI settings.

The first tool, RTITool, is aimed at performing all the preprocessing steps to transform acquired images to appearance profile array data cropped in the region of interest, prepared so that they can be used easily to estimate normals, 

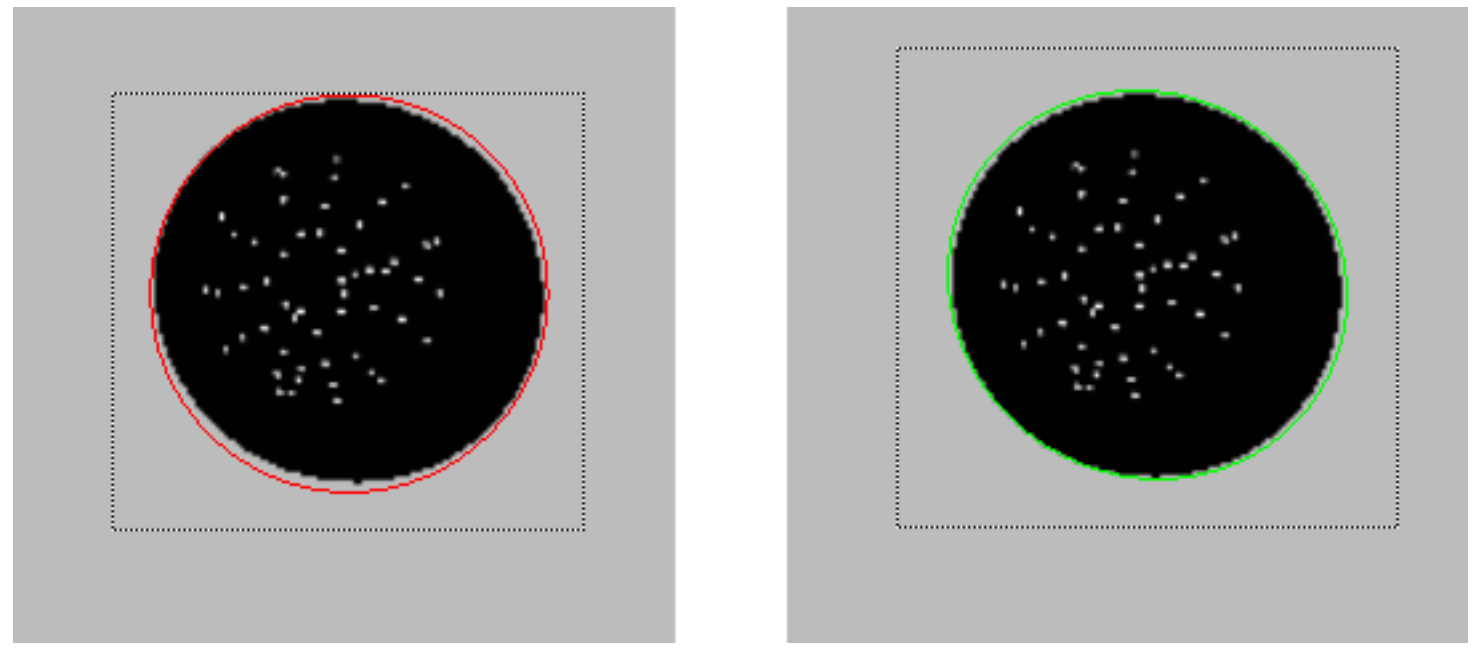

Figure 7: Annotation of reflective spheres on a synthetic dataset. With the orthographic assumption, automatic segmentation is not accurate and annotated circles cannot, in any case, match exactly the real object contours (left). With the perspective mode the segmentation is more accurate, and this results in a quite higher accuracy of the estimated light direction, as shown in Sec.5.1

relightable images and feature maps both with our own tools or other photometric stereo and RTI fitters. RTItool takes as input image and calibration information, and is able to perform all the calibration steps described in the previous section to cope with the difficulties of free-form acquisitions.

The second tool, APTool, is aimed at processing appearance profile array data using different algorithms, creating and exporting albedo, normal maps, relightable RTI files (e.g., PTM files), as well as displaying derived images (multilight image enhancements, relighted images from novel illumination directions) on a canvas window. Both tools are still a work in progress, but current versions, with all the capabilities described in the paper are available at the web site http://www.andreagiachetti.it/rtitools. Code has been developed on a Linux platform, but, as it has been realized in $\mathrm{C}++$ using Qt and OpenCV libraries, it could be easily ported on a variety of computing architectures.

\subsection{RTITool}

This program allows the user to load image sets, both trough image lists or through files with filenames and associated light directions typically used in current RTI tools. Users can then perform all the processing pipeline with various option, working with both 8-bit and 16-bit depth images and generating APA files for the entire image size or cropped regions. The interface is designed to simplify all the annotating tasks. For example, in order to easily annotate reflective spheres, annotation and automatic fitting algorithms are by default done on the maximum image, showing the highest luminance pixels over the stack, removing shadows and evidencing the black object (Fig. 3, left). In the same way, the annotation of the white frame is done by showing the minimum image, displaying the lowest per-pixel luminance, so as to easily avoid annotating regions that can be shadowed from some light directions (Fig. 3 , right).

The annotation of reflective spheres is semi-automatic. The user is asked to draw a rectangle including each sphere image. The circles (in case of orthographic assumption) or the ellipses (in case of perspective) are automatically estimated and drawn. Users can also visually refine the segmentation by interactively changing the curve parameters on the interface. Fig. 7 shows the inaccuracy of classical circular annotation (left), fixed on the same image by the ellipse fitting. In both cases, light directions can be estimated and stored. Note that even an apparently small deviation from the orthographic model, as the one shown in the figure, may result in an increase of one order of magnitude of error in light direction estimation (see Sec.5.1).

\subsection{APTool}

The processing of the raw MLIC stack performed with the RTITool ends by the storage of the data structure allowing the sequential processing of pixel information (light directions and associated corrected or non corrected 
intensity values). This information can be used to estimate normals and albedo using photometric stereo, creating novel relighted or enhanced images by interpolating or mixing the different pixel values, fitting reflectance models storing relightable images like PTM or HSH standard files, and more. We developed, for these purposes, a second software tool called APTool, which loads preprocessed arrays and allows the generation of normal and albedo maps derived from PS or the estimation of PTM coefficients. Robust versions of the fitters are also available. The idea is to include, in the future, different fitting and visualization algorithms to the software in order to support different kinds of end-user applications. Apart from fitting models and saving classical RTI files, the tool currently allows direct visualization of relighted images given a novel light direction, through direct interpolation of samples based on radial basis functions (Fig. 8). By selecting image locations (single points or rectangular regions), it is also possible to visualize a $2 \mathrm{D}$ intensity map represented in $l_{x}, l_{y}$ space of the local appearance profile, obtained by scattered data interpolation of the known samples (Fig. 8(b)). We have experimented with a Delaunay triangulation based and a Radial Basis Function interpolation of the samples, and provide an RBF implementation in the delivered tool.

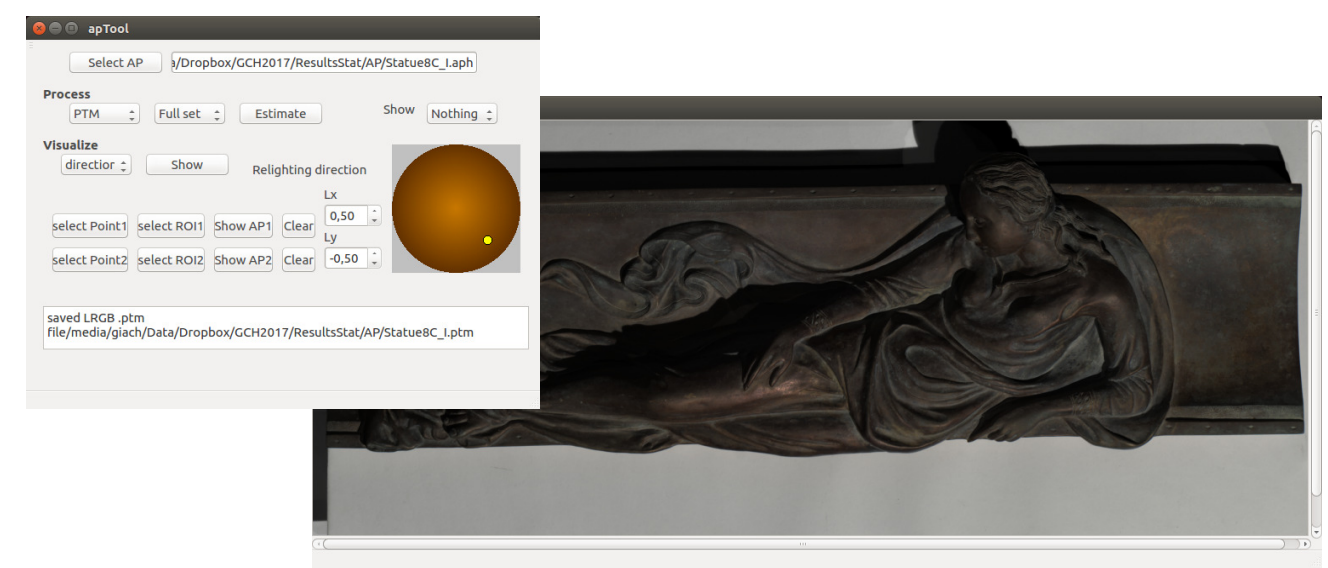

(a)

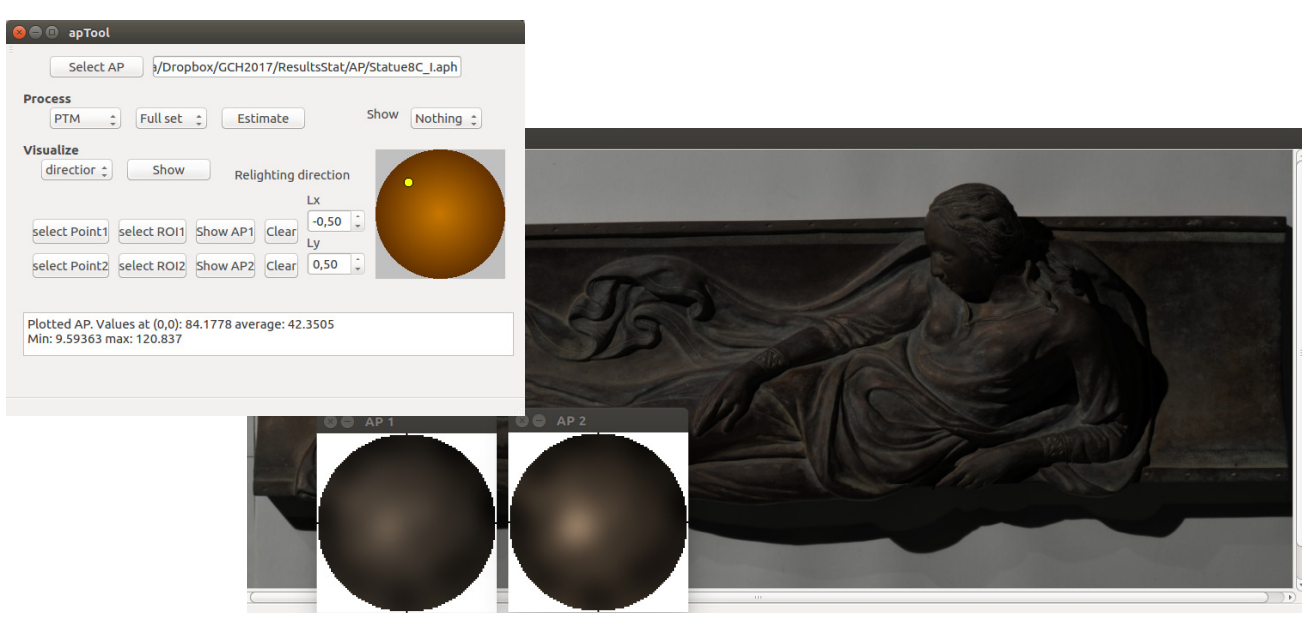

(b)

Figure 8: APTool used to show directly a Radial Basis function interpolation simulating direct relighting from $\left(l_{x}=0.5, l_{y}=-0.5\right)(a)$ and from $\left(l_{x}=-0.5, l_{y}=0.5\right)(\mathrm{b})$. In the second case, we also selected and compared AP profiles in selected image points.

\section{Experimental results}

In order to demonstrate the usability of our pipeline and the effects of new algorithms, we performed a series of experiments covering different kinds of MLIC capture and processing. Our tests include both synthetic datasets and 

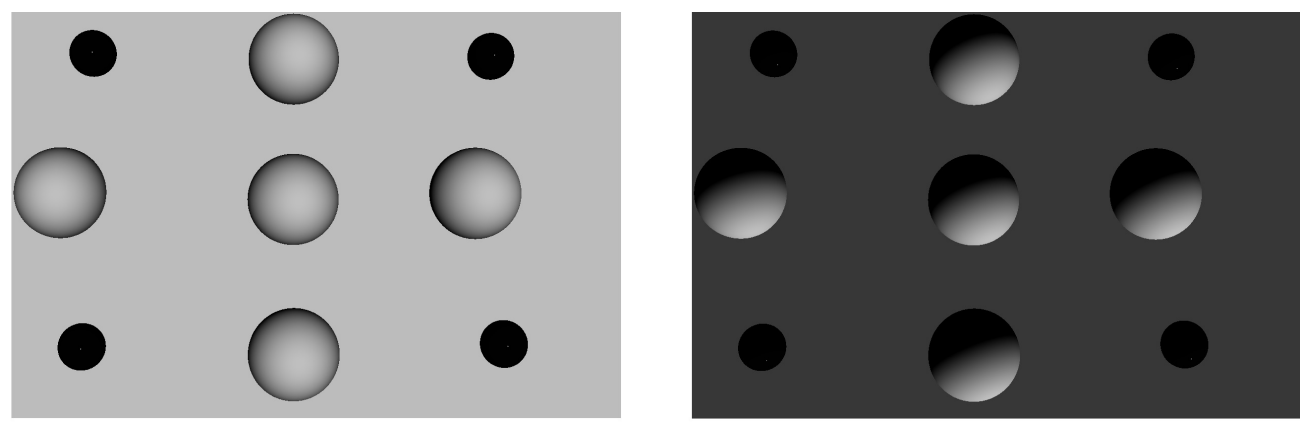

(a) Synthetic MLIC with directional lights
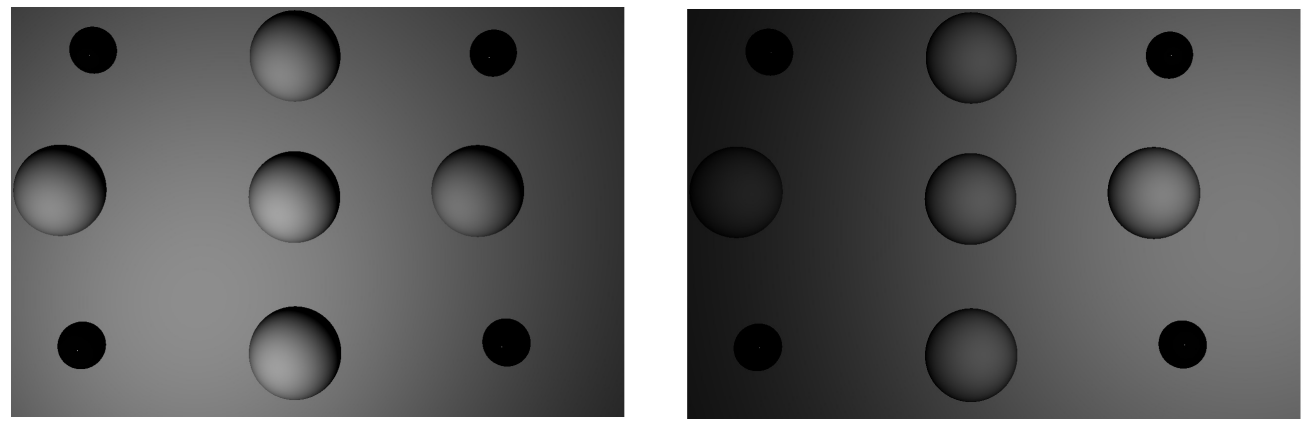

(b) Synthetic MLIC with spot lights

Figure 9: (a) Two images of a synthetic dataset simulating a white plane with some large bumps and 4 reflective spheres, acquired by a fixed camera under different parallel and constant illumination. (b) Two images of a synthetic dataset with the same geometry, but illuminated by simulated spot lights.

real-world ones. The real-world experiments were performed using a DSLR Nikon D810 camera with an architecture based on a CMOS sensor with removed IR cut-off filter. The size of the sensor is $36 \times 24 \mathrm{~mm}$ and the spatial resolution of the full format image area is 36MP. To the digital camera a full frame AF-S FX Nikkor 50mm f/1.8G lens was attached. As in this paper we present results using visible light, an IDAS-UIBAR III optical filter was used to gather only the signal from the visible range of the electromagnetic spectrum. The sensor of the digital camera was checked for linearity, by taking images covering a wide range of exposures, from very low to very high and then plotting the brightness as a function of exposure. The camera was geometrically calibrated by computing the intrinsic parameters, two radial and two tangential distortion coefficients with the GML Camera Calibration Toolbox. However, any other calibration tool can be used for this purpose.

\subsection{Accuracy of light direction estimation}

In order to evaluate the errors in light direction estimation when the orthographic camera model is not perfectly followed, we created a synthetic RTI dataset by rendering a scene with 4 reflective spheres near image corners, placed on top of a white Lambertian surface not exactly perpendicular to the camera axis, and with some spherical bumps, illuminated with perfectly parallel rays along 50 known directions (Fig. 9 a) or with the same number of simulated spot lights (Fig. 9 b). Using RTItool, we annotated the elliptic sphere profiles and estimated the light directions at each sphere position as described in Sec. 3.2. We compared the results with those obtained with our tool in the orthographic approximation, by annotating the circle circumscribed to the ellipse. We also compared our results with those obtained similarly with the widely used RTIBuilder package [21]. Note that the circular annotation cannot be precise, as the sphere mask is actually elliptic, as shown in Fig. 7, and this happens in most real images.

A comparison of the errors obtained (difference between the average of the four sphere estimations and ground truth) reveals that, despite the limited eccentricity of the ellipses, with the perspective model we have the error reduced by an order of magnitude. The average errors for the 50 directions of Fig. 9 a are, in fact, 0.02 radians for the per- 


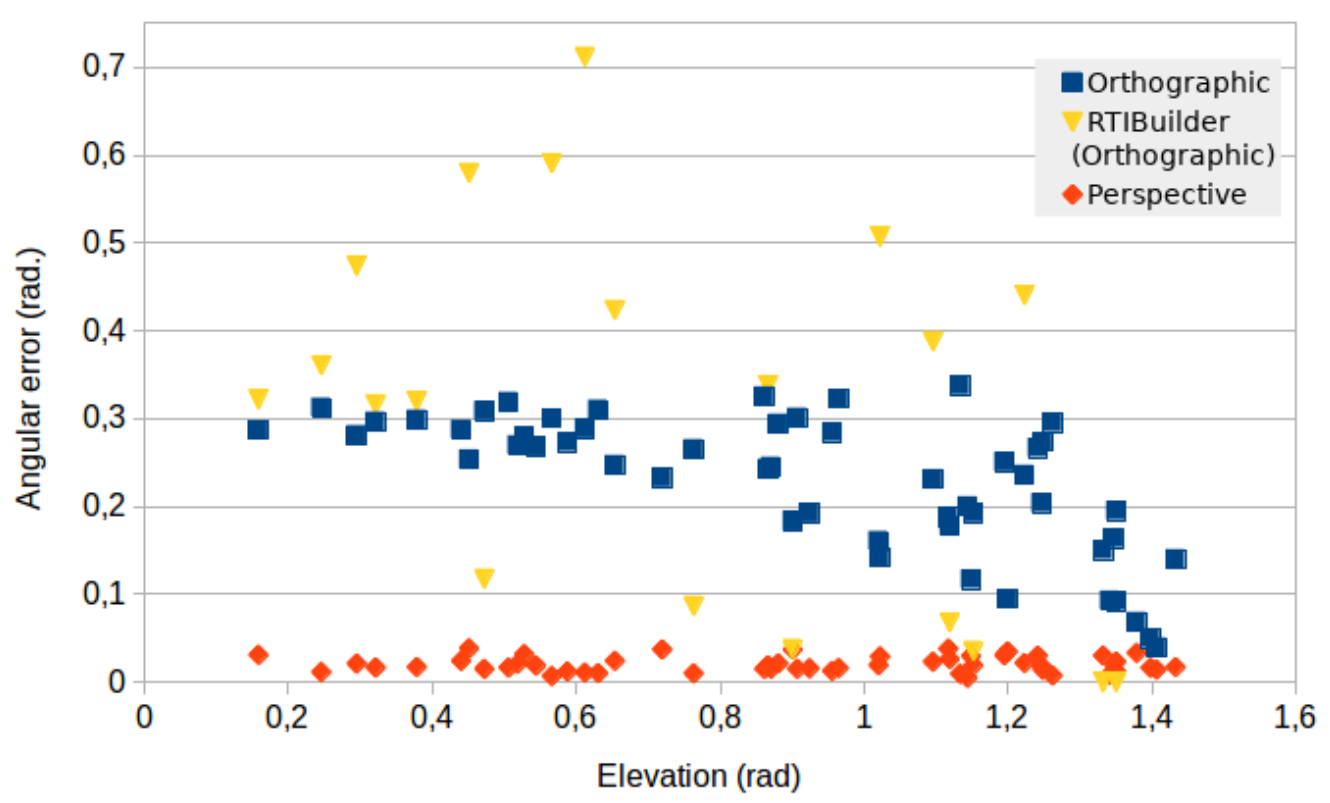

Figure 10: Angular errors due to wrong orthogonal camera assumption can be quite relevant. The use of a perspective model makes the values quite accurate.

spective estimation, 0.21 for the orthographic estimation done with RTITool, and 0.23 for the orthographic estimation done with RTIBuilder. Fig. 10 shows errors for each of the 50 single light directions sorted by elevation.

The local accuracy of light direction estimation is then improved by estimating local values by interpolating the values of the sphere at the corners. In order to show the amount of error reduction, we conducted two experiments. First, we created another synthetic dataset similar to the previous one, but where the images are illuminated with simulated spot lights approximately pointed towards the center of the target. Light direction and intensity for each pixel are thus not uniform, as in most typical real-world scenarios. In this case, the per pixel average error in the constant estimation (average of the values of the four spheres) is significantly higher than the error value coming from interpolation. Fig. 11 shows the average errors for the single images plotted versus elevation angle of the spotlight orientation. With interpolation, the error, averaged on all pixels of all images, is reduced from 0.17 to 0.05 degrees.

We also performed experiments on a real acquisition of a calibration target. In this case, we captured images of a flat plane perpendicular to the camera axis, putting four reflective spheres at the corners of the image area and a fifth in the center. In the set of images captured, the average difference between the direction measured in the top left corner and the one measured in the image center was 0.146 radians. Fig. 12 shows that, for small elevation angles, the error is higher due to the larger effect of quantization error in highlight localization. If we estimate a per-pixel linear interpolation of light direction, we reduce the average error to 0.121 radians.

\begin{tabular}{|lr|r|}
\cline { 2 - 3 } \multicolumn{1}{c|}{} & Angular err (rad) & Std. Dev \\
\hline Single Directions, no light correction & 0,482 & 0,271 \\
\hline Interpolated directions, no light correction & 0,417 & 0,249 \\
\hline Single Directions, light correction & 0,262 & 0,188 \\
\hline Interpolated directions, light correction & 0,252 & 0,183 \\
\hline
\end{tabular}

Table 1: Angular errors reduction in per-pixel normal estimation with Photometric Stereo on the synthetic "spotlight" dataset of Fig. 9 b using interpolated light direction estimation and intensity correction. 


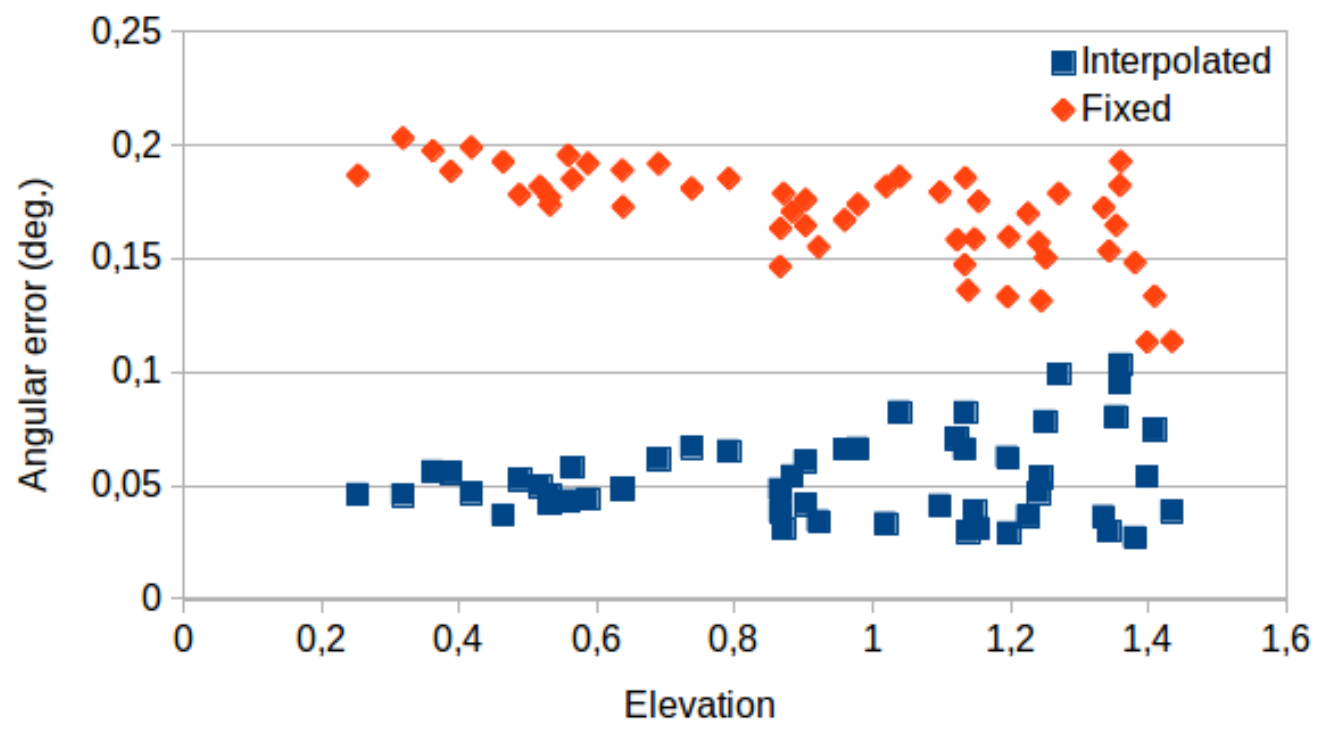

Figure 11: Angular errors of light direction estimated on a sphere with respect to real one in the simulated "spotlight" dataset of Fig. 9 b, plotted against elevation of the principal ground truth spotlight direction. Interpolation strongly increase the average accuracy of the per-pixel estimation.

\subsection{Accuracy of normal reconstruction}

The improved per-pixel light direction estimation and the procedure to correct illumination increase the quality of the MLIC-based reconstructions, as demonstrated, for instance, by our tests with Photometric Stereo and normal estimation. Using the same simulated dataset with spotlight illumination, we estimated surface normals (and albedo) by solving the classical least-squares problem under the assumption of Lambertian surfaces. We then compared perpixel reconstructed normals with the ground truth values. Table 5.1 shows that the average angular error is strongly reduced both by the light direction interpolation and the light intensity correction.

The effects of light correction can be also appreciated when reconstructing normals of challenging real-world objects from MLICs using Photometric Stereo. To show this, we acquired images of a set of coins placed over a flat background. We used our reconstruction pipeline and the RTITool to recover the appearance profile arrays and then used our APTool to reconstruct normal maps.

The set is composed by a bronze Roman coin (quadrans) dated 9 B.C. and damaged by scratches, and two 10 cent Italian coins. One exemplar, dated 1931 is made of copper and is severely degraded, while the second exemplar, dated 1939, is made of a special alloy with nickel, called Bronzital, which has been used to improve corrosion resistance.

Normal maps obtained with Photometric Stereo have been compared with an (approximate) reference solution derived from a high resolution $3 \mathrm{D}$ reconstruction of the same coins made with an optical microprofilometer based on conoscopic holography [44]. This device is able to capture reliable profilometric measurements down to the scale of micron on different kinds of materials, reflective or diffusive. Our microprofilometer is based on an Optimet conoscopic probe mounted on sub-micrometric linear stages in order to scan a region up to $30 \times 30 \mathrm{~cm}^{2}$ in one session. Reference coin models have been reconstructed with a transversal resolution (XY grid) of 50 microns.

Depth maps derived from these models were finally registered with the estimated RTI normal maps using a similarity transform optimized to match the correspondence of manually selected points (12 landmarks). This initial registration was then refined by locally optimizing mutual information in image space.

Fig. 13 shows the three coins and the related differences between RTI-based normal maps and the reference normal maps estimated from microprofilometric data, both in case of non-corrected image brightness, and with the light correction procedure described in Sec. 3.4. It is evident that light correction sensibly improves the reconstruction quality, as quantitatively reported in Table 5.2. The light correction procedure reduces the median errors, on average, by $27 \%$. 


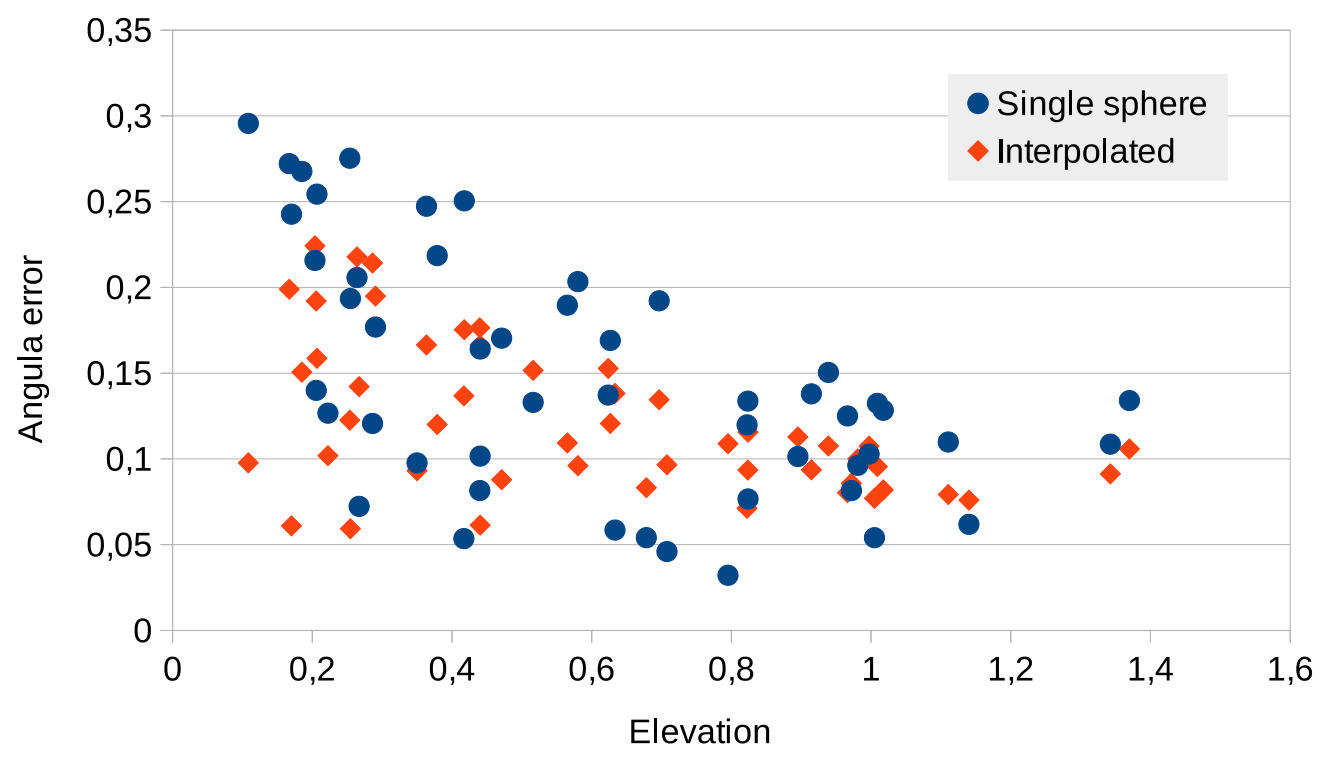

Figure 12: Angular difference of the direction estimated on a sphere near the corner with respect to the one estimate on a sphere near the image center (blue dots), plotted as a function of elevation for a complete MLIC scan (50 photos). Replacing the corner estimate with the linear interpolation of the four corner values in the central position, we can get reduced errors (red squares).

\begin{tabular}{|l|r|r|}
\cline { 2 - 3 } \multicolumn{1}{c|}{} & \multicolumn{2}{c|}{ Median angular distance (rad.) } \\
\cline { 2 - 3 } \multicolumn{1}{c|}{} & Non-corrected & Corrected \\
\hline Bronzital 10c & 0.117 & 0.079 \\
\hline Copper 10c & 0.068 & 0.053 \\
\hline Quadrans & 0.171 & 0.108 \\
\hline
\end{tabular}

Table 2: Median angular distances of the RTI estimated normals from the reference microprofilometer normals. The calibration procedure reduces the errors on average of 27 percent.

\subsection{Recovery of reflectance properties of materials}

Our intensity correction methods are also important to better recover material properties. To demonstrate this fact, we placed a matte paper target with different albedo regions in different positions on a flat planar background (Fig. 14).

If we visualize the interpolated appearance profiles estimated on a pixel in a selected region, in this case with flat perpendicular surface and approximately Lambertian behavior, we should see a function that, represented in $l_{x}, l_{y}$ coordinates, should present a regular and symmetric function. Fig. 15 shows that plots of interpolated appearance profiles on non-corrected images are not symmetric and different in different regions of the same material if images are not corrected with our procedure. Conversely, light correction results in profiles similar to those expected and similar in different parts of the image where the material is the same.

This effect can be quantitatively measured by evaluating the average albedo of the patches of the same paper types put in two different positions in the scene of Fig. 14. Without corrections, the albedo of paper patches of the same type placed in different image position differs up to 7\%. The difference is strongly reduced with the light direction and intensity correction procedure, as shown in Table 5.3.

By matching the reflectance of the Lambertian frame to a reference value, we can also estimate the consistency of albedo measurements among different image captures. Table 5.3 shows that the measurements obtained in a second acquisition are largely different (often more than $30 \%$ of the value), even if a similar protocol and the same light source has been used. It is actually sufficient to change the distance of the source to have different results. However, the use of the correction procedure results in similar albedo values (difference lower than $2.5 \%$ of the value) 

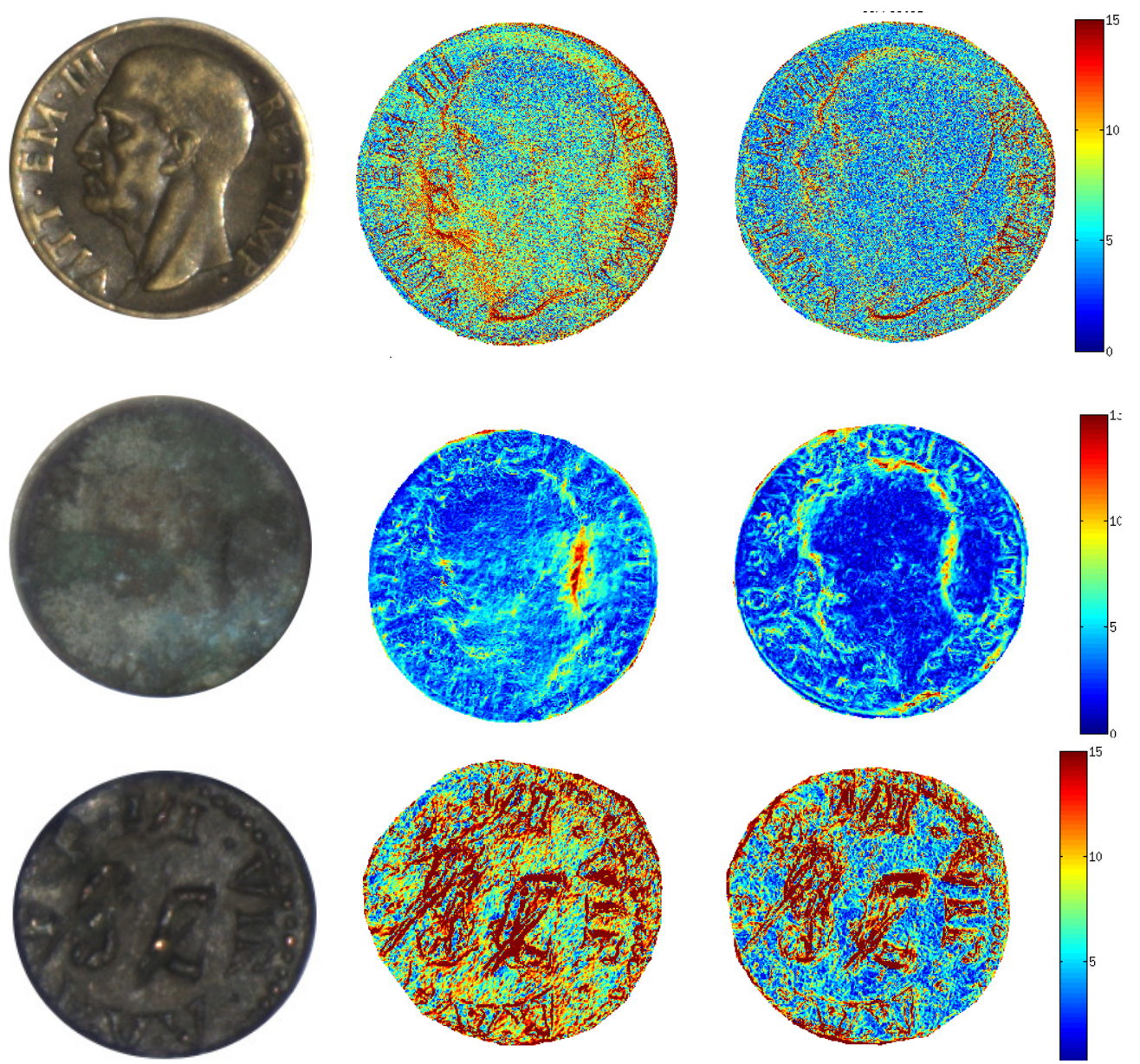

Figure 13: Angular errors. Color-coded angular errors (degree) of RTI estimated normals wrt ground truth from microprofilometry. Left: noncalibrated results. Right: results with light calibration.

Another important effect of the light correction procedure is the repeatability of reflectance parameters estimation in different MLIC captures without light calibration. Table 5.3 shows the albedo of the same paper types of the previous experiment estimated on a different MLIC capture of a plane with paper targets glued on it. Without light correction, the light intensity is quite different, even if we tried to use a similar configuration. Clearly a small difference in light distance results in different illumination and estimated albedo. The light correction procedure, by contrast, makes the estimated parameters similar.

Processed RTI data is often used to segment different materials not easily recognized in color images [18]. Such kind of results can be improved by our light correction procedures. To show this, we have performed two RTI acquisitions of a polished silver sample partially covered by a coating, see Fig. 16(a), and applied unsupervised classification to segment regions with or without coating. For each RTI sample, we compute a 7-dimensional descriptor of a 30 pixels neighborhood. The descriptor is the average albedo value, to account for material color, plus the 6 standard deviations of the standard RTI polynomial coefficients, to account for the roughness of the sample surface.

Unsupervised classification is achieved by performing two-class k-means clustering. We measured the similarity of the classification outcomes obtained from the two different acquisitions, without and with light calibration, see Fig. 16. The only difference between the two acquisitions of the same sample is the different lighting pattern caused by the free-form approach. The coated area is in red, while the uncoated area is in green. In the absence of light calibration, the clustering outcome is unstable, as it has only a $20 \%$ overlap, while, by performing light calibration, 

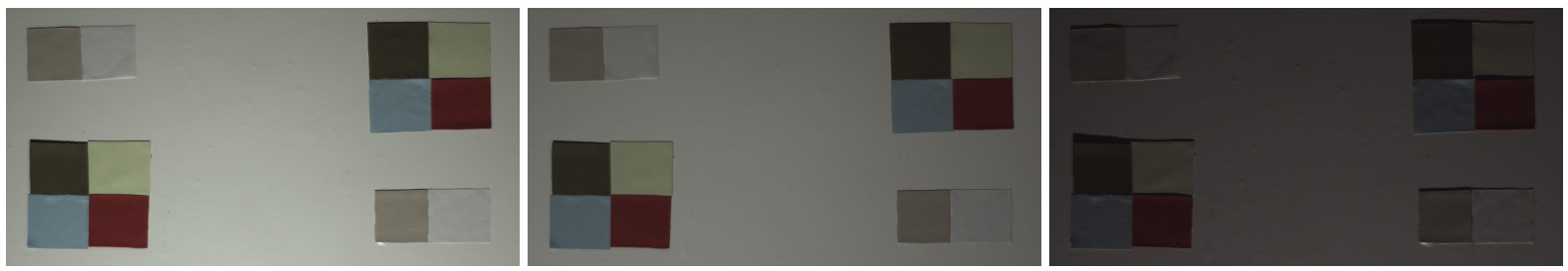

Figure 14: Three images of a MLIC capture of a planar surface with flat paper targets with different albedo regions.

\begin{tabular}{|l|l|r|c|r|c|r|}
\cline { 3 - 7 } \multicolumn{2}{c|}{} & $\begin{array}{c}\text { Albedo } \\
\text { Pos. 1 }\end{array}$ & std & $\begin{array}{c}\text { Albedo } \\
\text { Pos.2 }\end{array}$ & std & diff/mean \\
\hline \multirow{2}{*}{ Paper1 } & corrected & 0,557 & 0,003 & 0,559 & 0,004 & $0,39 \%$ \\
\cline { 2 - 7 } & non-corrected & 0,726 & 0,009 & 0,708 & 0,009 & $2,53 \%$ \\
\hline \multirow{2}{*}{ Paper2 } & corrected & 0,406 & 0,003 & 0,411 & 0,003 & $1,38 \%$ \\
\cline { 2 - 7 } & non-corrected & 0,515 & 0,007 & 0,539 & 0,018 & $4,54 \%$ \\
\hline \multirow{2}{*}{ Paper3 } & corrected & 0,391 & 0,003 & 0,391 & 0,005 & $0,17 \%$ \\
\cline { 2 - 7 } & non-corrected & 0,501 & 0,009 & 0,514 & 0,007 & $2,73 \%$ \\
\hline \multirow{2}{*}{ Paper4 } & corrected & 0,157 & 0,004 & 0,156 & 0,003 & $0,55 \%$ \\
\cline { 2 - 7 } & non-corrected & 0,206 & 0,005 & 0,203 & 0,006 & $1,30 \%$ \\
\hline \multirow{2}{*}{ Paper5 } & corrected & 0,551 & 0,003 & 0,545 & 0,005 & $1,08 \%$ \\
\cline { 2 - 7 } & non-corrected & 0,734 & 0,008 & 0,685 & 0,010 & $6,96 \%$ \\
\hline \multirow{2}{*}{ Paper6 } & corrected & 0,138 & 0,006 & 0,135 & 0,003 & $2,10 \%$ \\
\cline { 2 - 7 } & non-corrected & 0,184 & 0,004 & 0,173 & 0,007 & $6,18 \%$ \\
\hline
\end{tabular}

Table 3: Albedo measured on planar patches of the same material can be quite different in different image regions if estimated with classic photometric stereo on non-corrected multi-light image stacks. Our brightness and light-direction correction procedures clearly result in more consistent values.

we improve it up to $99.5 \%$ of pixels that have been assigned to the same class, showing that with our approach free-form RTI can be used for surface characterization.

\subsection{Visual analysis of RTI enhancements}

The typical use of MLIC data done in the Cultural Heritage domain consists in estimating relightable images and analyzing them to improve the visualization of object details. To simulate this application, we created a mockup of a complex structure with fine relief details by imprinting a leaf on modeling paste, then acquiring the photos with our pipeline supporting light correction. We exported both corrected and non corrected appearance profiles with RTITool and estimated and exported PTM files with APTool. The files have been analyzed with RTIViewer to visualize interesting detail [21]. Fig. 17 shows a detail of a relighted image with the specular enhancement proposed in [10]. The result on top right is obtained from the non-corrected data, while the one on the bottom right is obtained with the corrected pipeline. In the corrected images, it is possible to appreciate a better enhancement of detail and a clearer visualization of nervatures and scratches, hardly visible on the uncorrected image.

This effect is even more visible in the example of Fig. 18, where specular enhancements obtained from PTM fitting of the non-corrected and corrected appearance profiles derived from the acquisition shown in Fig. 2(b) are compared. The correction leads to a much better visualization of brush strokes.

Looking at PTM-based relighting, it is interesting to note that even if light calibration ensure a better quality of enhancements due to the improved normals, the removal of specular components results in loss of possibly relevant information about the imaged object. This can be seen comparing relighted PTMs with corresponding relighted APA visualized with our tool. Fig.19 shows this on the painting detail. PTM-based relighting represents similarly regions where the surface has different specular behavior and the perception of depth is reduced by the absence of specular effects, visible in RBF interpolation.

We plan, therefore, to investigate on possible improvements of interactive direct visualization of APA information and on the development of novel enhancement methods that can be directly implemented in the APTool to allow 


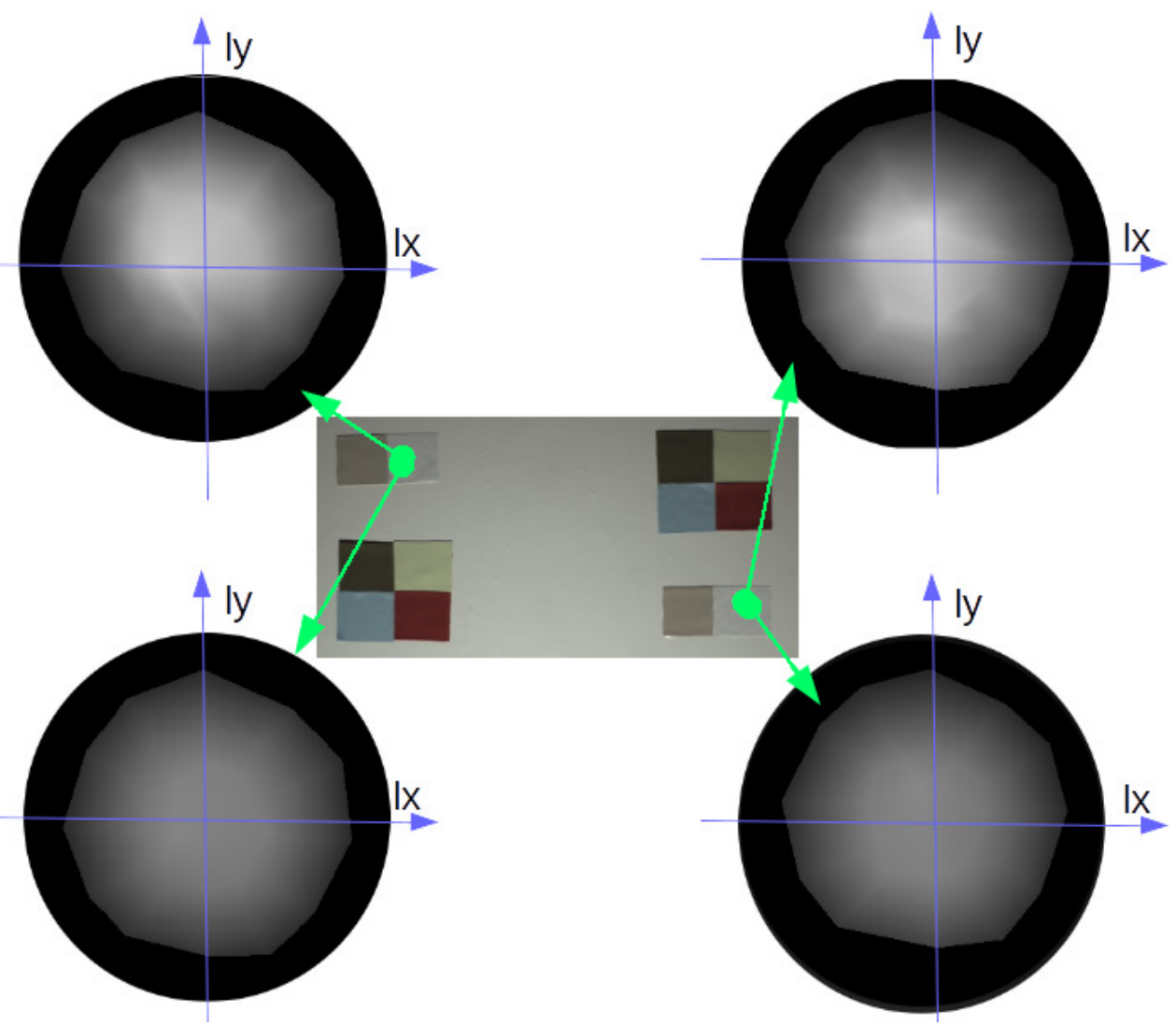

Figure 15: Appearance profiles corresponding to the same material on flat patches are similar and regularly shaped when computed on lightcorrected images (bottom), while are irregular when lighting is not uniformed (top)

a better visual analysis of the information hidden in RTI stacks. PTM or HSH encodings are useful as they allow compact storage of relightable images, but, imposing a drastically simplified reflectance model discarding relevant information, they may result in information loss that may create serious problems to the subsequent surface analysis. Our plan is to use smart compression techniques to obtain a compact representation of the full APA information allowing an easier handling and more efficient direct visualization.

\section{Discussion}

Highlight RTI is quite popular, especially in the Cultural Heritage domain, to the point that it may be considered one of the most successful computational photography techniques in that domain. It can be realized with a simple camera, a simple light source and one (or more) reflective spheres. However, the framework commonly used for this task has some limitations, and this can result in a low degree of repeatability of measurements, as well as in a poor quality of extracted information, leading, in some cases, to the impossibility of effectively using the technique.

In this article, we have shown that, with slight modifications of the standard acquisition setup, it is possible to significantly improve the quality of the fusion of a multi-light image collection, achieving a better reconstruction of shape and material properties of the scene, as well as an improved quality of relightable images. Our approach realizes a sort of integration of the classic H-RTI technique, usually based on uncalibrated lights and qualitative analysis, with the Material Capture and Photometric Stereo approaches targeted at accurate shape (and reflectance) reconstruction, but usually requiring very high-density acquisitions and/or light and camera calibration.

As with all practical setups, the proposed approach has also some limitations. First of all, the necessity of placing more targets near the object, and the fact that we assume that the object to be imaged is mostly planar. The latter 


\begin{tabular}{|l|l|r|r|r|}
\cline { 3 - 5 } \multicolumn{2}{c|}{} & $\begin{array}{r}\text { Albedo } \\
\text { MLIC 1 }\end{array}$ & $\begin{array}{r}\text { Albedo } \\
\text { MLIC 2 }\end{array}$ & diff/mean \\
\hline \multirow{2}{*}{ Paper1 } & corrected & 0,558 & 0,548 & $1,81 \%$ \\
\cline { 2 - 5 } & non-corrected & 0,717 & 0,518 & $32,25 \%$ \\
\hline \multirow{2}{*}{ Paper2 2} & corrected & 0,409 & 0,404 & $1,18 \%$ \\
\cline { 2 - 5 } & non-corrected & 0,527 & 0,393 & $29,23 \%$ \\
\hline \multirow{2}{*}{ Paper33 3} & corrected & 0,391 & 0,385 & $1,60 \%$ \\
\cline { 2 - 5 } & non-corrected & 0,508 & 0,381 & $28,45 \%$ \\
\hline \multirow{2}{*}{ Paper4 } & corrected & 0,156 & 0,156 & $0,23 \%$ \\
\cline { 2 - 5 } & non-corrected & 0,205 & 0,155 & $27,64 \%$ \\
\hline \multirow{2}{*}{ Paper5 } & corrected & 0,548 & 0,536 & $2,22 \%$ \\
\cline { 2 - 5 } & non-corrected & 0,710 & 0,510 & $32,77 \%$ \\
\hline \multirow{2}{*}{ Paper6 } & corrected & 0,136 & 0,139 & $2,06 \%$ \\
\cline { 2 - 5 } & non-corrected & 0,178 & 0,135 & $27,84 \%$ \\
\hline
\end{tabular}

Table 4: Albedo values measured on a different acquisition of the same material patches of Table 3 . The correction procedure results in similar values for similar materials.

assumption is, however, typically true in H-RTI applications, and can be resolved with the same iterative techniques applied in PS settings. Moreover, in our current implementation, using our custom designed frame with the four spheres and the coated aluminum bars, the size of the object to be captured is limited to a range from about $50 \times 50 \mathrm{~cm}$ to $1 \mathrm{mx} 1 \mathrm{~m}$. For larger sizes, the placement of co-planar Lambertian targets to estimate the correction may be difficult in on-site acquisitions. We are investigating, however, different light correction methods that may take into account depth variations of the illumination. We are also investigating improved interpolation methods tuned for standard spot lights.

Our current work focuses on the finalization and testing of our processing tools, that will be freely available for the scientific community.

We are also investigating novel techniques for shape and material reconstruction, as well for feature detection from MLIC. A challenging problem is, for example, the development of robust fitting techniques able to recover material reflectance information independently from shape. Apart from the difficulty in modeling reflectance, releasing hypotheses of Lambertian behavior, it is also necessary to consider that pixel information is not always depending only on local shape and reflectance, but also to global effects like inter-reflections and projected shadows. The use of classic outlier removal procedures, proposed in previous works [43], may be problematic due to the relatively low number of samples and more specific heuristics for outlier rejection may be more effective.

\section{Acknowledgments}

This work was partially supported by the Scan4Reco project funded by European Union's Horizon 2020 Framework Programme for Research and Innovation under grant agreement no 665091 and by the DSURF project (PRIN 2015) funded by the Italian Ministry of University and Research. We also acknowledge the contribution of Sardinian Regional Authorities under projects VIGEC and Vis\&VideoLab. The authors wish to thank Opificio Delle Pietre Dure, Vittorio Barra and Alberto Dal Chiele and for providing the artworks shown in our experiments.

\section{References}

[1] R. Basri, D. Jacobs, I. Kemelmacher, Photometric stereo with general, unknown lighting, International Journal of Computer Vision 72 (2007) $239-257$.

[2] C.-H. Hung, T.-P. Wu, Y. Matsushita, L. Xu, J. Jia, C.-K. Tang, Photometric stereo in the wild, in: 2015 IEEE Winter Conference on Applications of Computer Vision, IEEE, 2015, pp. 302-309.

[3] M. Mudge, T. Malzbender, C. Schroer, M. Lum, New reflection transformation imaging methods for rock art and multiple-viewpoint display., in: VAST, volume 6, Citeseer, 2006, pp. 195-202. 


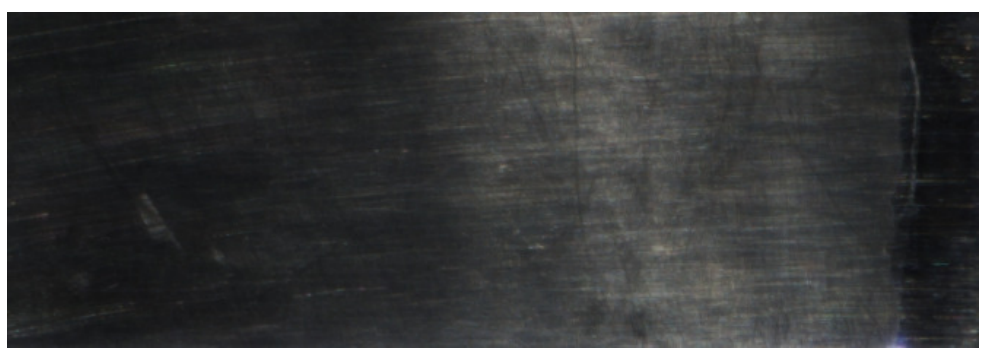

(a) Partially coated silver sample
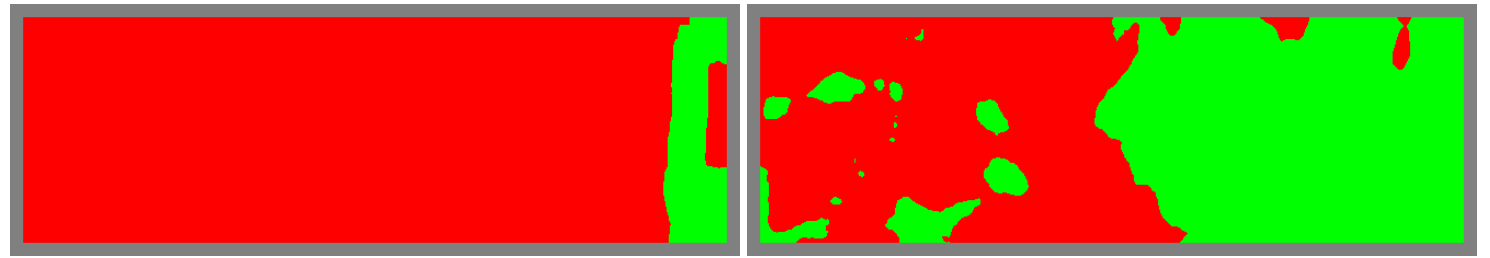

(b) Clustering results without light correction
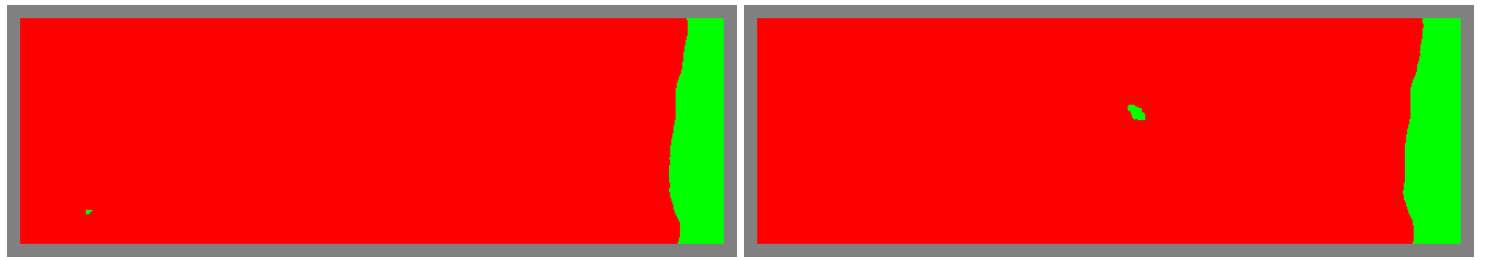

(c) Clustering results with light correction

Figure 16: Unsupervised classification. In (b),(c), two class k-means clustering (left/right) applied to two different acquisitions of the polished sample in (a) are represented. The coated area is in red while the uncoated area is in green. Without calibration we have a) $20 \%$ of classification similarity, while we obtain a value of $99.5 \%$ by using the calibrated images. This shows the drastically increased level of repeatability of the proposed pipeline with respect to classic free-form RTI.

[4] A. Duliu, R. Brosig, S. Ognawala, T. Lasser, M. Ziai, N. Navab, Illumination compensation and normalization using low-rank decomposition of multispectral images in dermatology, in: International Conference on Information Processing in Medical Imaging, Springer, 2015, pp. $613-625$.

[5] N. J. Durr, G. González, D. Lim, G. Traverso, N. S. Nishioka, B. J. Vakoc, V. Parot, System for clinical photometric stereo endoscopy, in: SPIE BiOS, International Society for Optics and Photonics, 2014, pp. 89351F-89351F.

[6] M. Massot-Campos, G. Oliver-Codina, Optical sensors and methods for underwater 3d reconstruction, Sensors 15 (2015) $31525-31557$.

[7] J. Ackermann, M. Goesele, A survey of photometric stereo techniques, Foundations and Trends in Computer Graphics and Vision 9 (2015) $149-254$.

[8] R. J. Woodham, Photometric stereo: A reflectance map technique for determining surface orientation from image intensity, in: 22nd Annual Technical Symposium, International Society for Optics and Photonics, 1979, pp. 136-143.

[9] R. J. Woodham, Photometric method for determining surface orientation from multiple images, Optical engineering 19 (1980) 191139.

[10] T. Malzbender, D. Gelb, H. Wolters, Polynomial texture maps, in: Proceedings of the 28th annual conference on Computer graphics and interactive techniques, ACM, 2001, pp. 519-528.

[11] M. Mudge, J.-P. Voutaz, C. Schroer, M. Lum, Reflection transformation imaging and virtual representations of coins from the hospice of the grand st. bernard, in: Proceedings of the 6th International Conference on Virtual Reality, Archaeology and Intelligent Cultural Heritage, VAST'05, Eurographics Association, Aire-la-Ville, Switzerland, Switzerland, 2005, pp. 29-39. URL: http://dx.doi.org/10.2312/VAST/VAST05/029-039. doi:10.2312/VAST/VAST05/029-039.

[12] P. Gautron, J. Krivanek, S. N. Pattanaik, K. Bouatouch, A novel hemispherical basis for accurate and efficient rendering., Rendering Techniques 2004 (2004) 321-330.

[13] G. Pitard, G. Le Goïc, H. Favrelière, S. Samper, S.-F. Desage, M. Pillet, Discrete modal decomposition for surface appearance modelling and rendering, in: SPIE Optical Metrology, International Society for Optics and Photonics, 2015, pp. 952523-952523.

[14] R. Raskar, K.-H. Tan, R. Feris, J. Yu, M. Turk, Non-photorealistic camera: depth edge detection and stylized rendering using multi-flash imaging, in: ACM transactions on graphics (TOG), volume 23, ACM, 2004, pp. 679-688.

[15] R. Fattal, M. Agrawala, S. Rusinkiewicz, Multiscale shape and detail enhancement from multi-light image collections, ACM Trans. Graph. 26 (2007) 51

[16] C. Brognara, M. Corsini, M. Dellepiane, A. Giachetti, Edge detection on polynomial texture maps, in: Image Analysis and Processing-ICIAP 

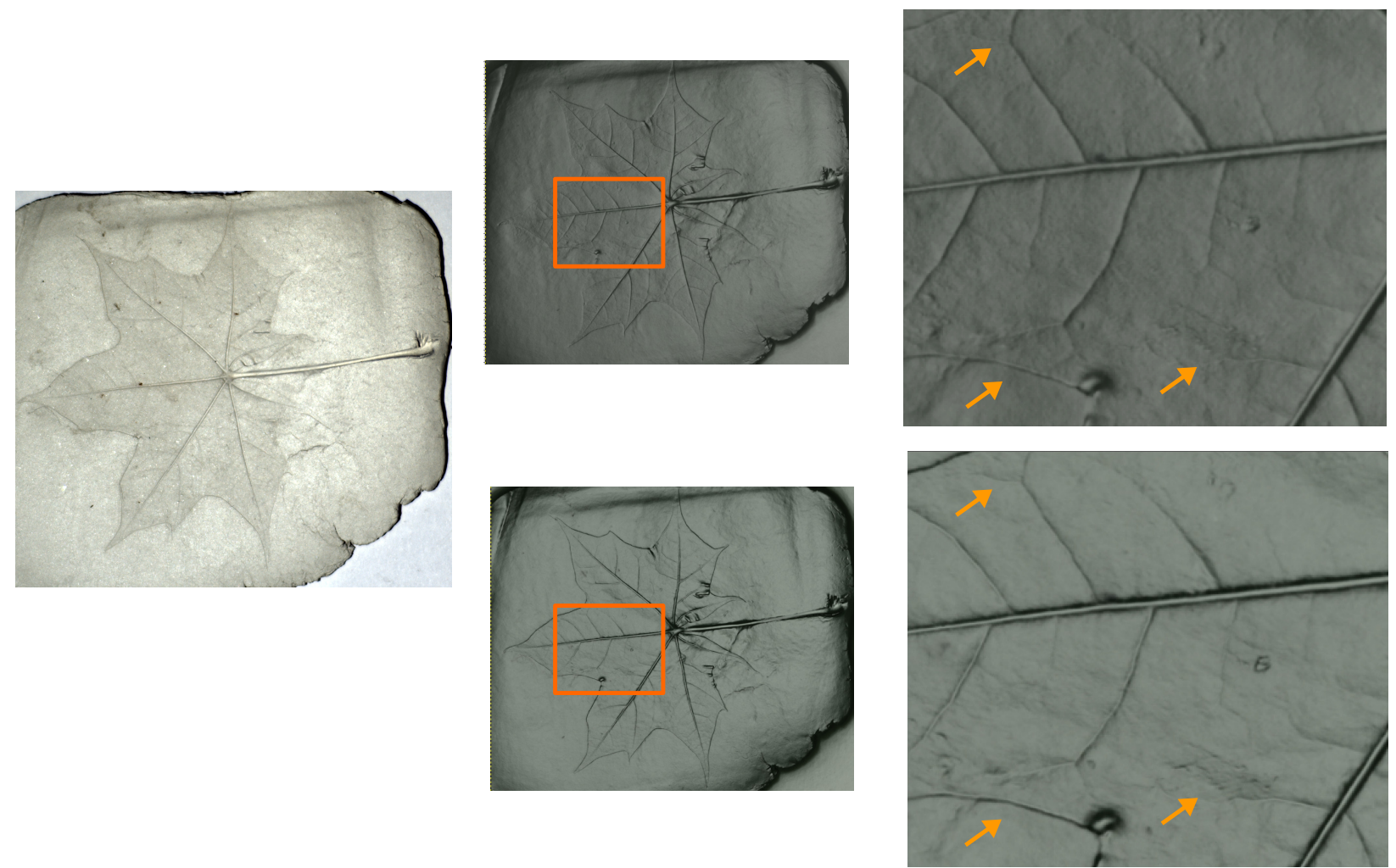

Figure 17: Detail of relighting with specular enhancements of a captured mock up representing a leaf with small imprinted details. Using RTIViewer with the same parameters, the result obtained with the PTM files estimated using corrected images (bottom) allows a better perception of small details.

2013, Springer, 2013, pp. 482-491.

[17] R. Pan, Detection of edges from polynomial texture maps, 3D Research 7 (2016) 1-8.

[18] O. Wang, P. Gunawardane, S. Scher, J. Davis, Material classification using brdf slices, in: Computer Vision and Pattern Recognition, 2009. CVPR 2009. IEEE Conference on, IEEE, 2009, pp. 2805-2811.

[19] C. R. Johnson, P. Messier, W. A. Sethares, A. G. Klein, C. Brown, A. H. Do, P. Klausmeyer, P. Abry, S. Jaffard, H. Wendt, et al., Pursuing automated classification of historic photographic papers from raking light images, Journal of the American Institute for Conservation 53 (2014) 159-170.

[20] I. M. Ciortan, R. Pintus, G. Marchioro, C. Daffara, A. Giachetti, E. Gobbetti, A Practical Reflectance Transformation Imaging Pipeline for Surface Characterization in Cultural Heritage, in: C. E. Catalano, L. D. Luca (Eds.), Eurographics Workshop on Graphics and Cultural Heritage, The Eurographics Association, 2016. doi:10.2312/gch.20161396.

[21] CHI, Cultural heritage imaging, 2016. URL: http://culturalheritageimaging.org.

[22] R. Szeliski, Computer vision: algorithms and applications, Springer Science \& Business Media, 2010.

[23] J. Dorsey, H. Rushmeier, F. Sillion, Digital modeling of material appearance, Morgan Kaufmann, 2010.

[24] R. Pintus, K. Pal, Y. Yang, T. Weyrich, E. Gobbetti, H. Rushmeier, A survey of geometric analysis in cultural heritage, in: Computer Graphics Forum, volume 35, Wiley Online Library, 2016, pp. 4-31.

[25] C. Schwartz, R. Sarlette, M. Weinmann, R. Klein, Dome ii: A parallelized btf acquisition system, in: Proceedings of the Eurographics 2013 Workshop on Material Appearance Modeling: Issues and Acquisition, Eurographics Association, 2013, pp. 25-31.

[26] H. Hameeuw, Mesopotamian clay cones in the ancient near east collections of the royal museums of art and history, Bulletin van de Koninklijke Musea voor Kunst en Geschiedenis 84 (2015) 5-48.

[27] C. Liu, J. Gu, Discriminative illumination: Per-pixel classification of raw materials based on optimal projections of spectral brdf, Pattern Analysis and Machine Intelligence, IEEE Transactions on 36 (2014) 86-98.

[28] L. Watteeuw, H. Hameeuw, B. Vandermeulen, A. Van der Perre, V. Boschloos, L. Delvaux, M. Proesmans, M. Van Bos, L. Van Gool, Light, shadows and surface characteristics: the multispectral portable light dome, Applied Physics A 122 (2016) 976.

[29] W. Xie, C. Dai, C. C. Wang, Photometric stereo with near point lighting: A solution by mesh deformation, in: Computer Vision and Pattern Recognition (CVPR), 2015 IEEE Conference on, IEEE, 2015, pp. 4585-4593.

[30] T. Papadhimitri, P. Favaro, Uncalibrated near-light photometric stereo, in: Proceedings of the British Machine Vision Conference, BMVA Press, 2014. doi:http://dx.doi.org/10.5244/C.28.128.

[31] J. Ahmad, J. Sun, L. Smith, M. Smith, An improved photometric stereo through distance estimation and light vector optimization from 


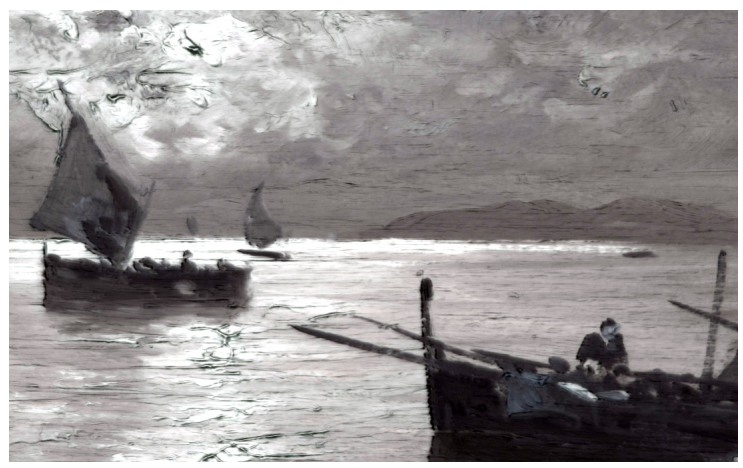

(a) Non corrected

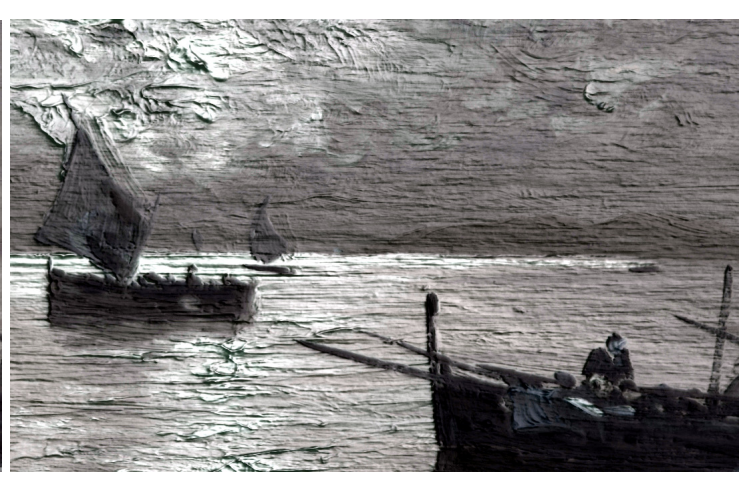

(b) Corrected

Figure 18: Relighting with $l_{x}=0, l_{y}=0$ and specular enhancement of PTMs estimated on the non-corrected appearance profile array (a) and corrected appearance profile array (b) coming from the acquisition of Fig. 2. The second one shows much better the relief of the brush strokes and the painting style.

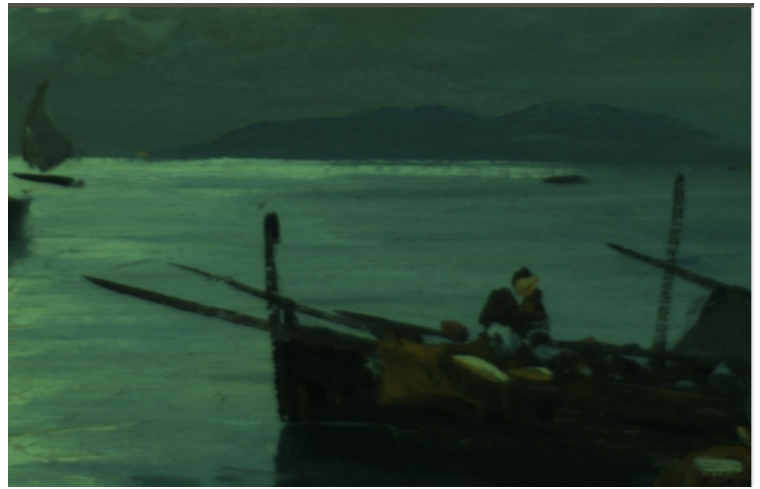

(a)

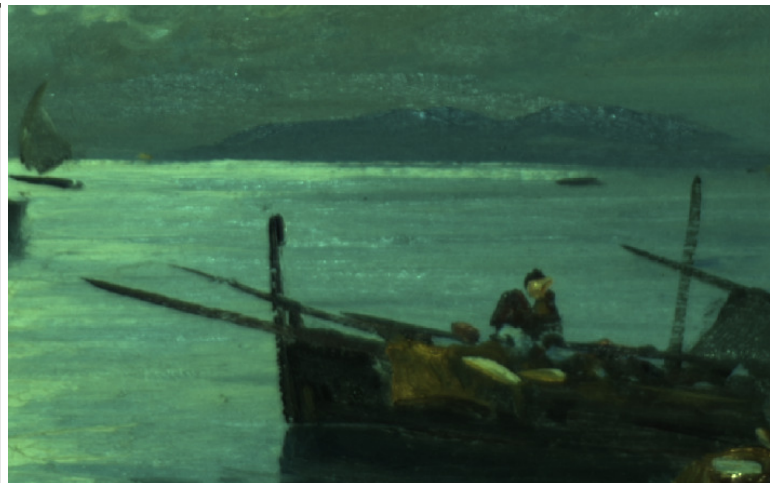

(b)

Figure 19: Painting details relighted from the same direction $\left(l_{x}=0, l_{y}=0\right)$ using (a) interpolation based on PTM coefficients (b) Radial Basis Functions interpolation on APA data. Material differences and relief details are not visible on the PTM visualization while are correctly perceived in the direct relighting.

diffused maxima region, Pattern Recognition Letters 50 (2014) 15-22.

[32] H. Ukida, Y. Tanimoto, T. Sano, H. Yamamoto, 3d shape and color estimation using linear light sources and cameras, in: Imaging Systems and Techniques (IST), 2015 IEEE International Conference on, IEEE, 2015, pp. 1-5.

[33] M. Corsini, M. Callieri, P. Cignoni, Stereo light probe, in: Computer Graphics Forum, volume 27, Wiley Online Library, 2008, pp. 291-300.

[34] J. Ackermann, S. Fuhrmann, M. Goesele, Geometric Point Light Source Calibration, in: M. Bronstein, J. Favre, K. Hormann (Eds.), Vision, Modeling \& Visualization, The Eurographics Association, 2013. doi:10.2312/PE.VMV.VMV13.161-168.

[35] R. Pintus, I. M. Ciortan, A. Giachetti, E. Gobbetti, Practical Free-form RTI Acquisition with Local Spot Lights, in: G. Pintore, F. Stanco (Eds.), Smart Tools and Apps for Graphics - Eurographics Italian Chapter Conference, The Eurographics Association, 2016. doi:10.2312/stag.20161374.

[36] L. Xie, Z. Song, G. Jiao, X. Huang, K. Jia, A practical means for calibrating an led-based photometric stereo system, Optics and Lasers in Engineering 64 (2015) $42-50$

[37] J. Sun, M. Smith, L. Smith, A. Farooq, Sampling light field for photometric stereo, International Journal of Computer Theory and Engineering 5 (2013) 14.

[38] M. E. Angelopoulou, M. Petrou, Uncalibrated flatfielding and illumination vector estimationfor photometric stereo face reconstruction, Machine vision and applications 25 (2014) 1317-1332.

[39] A. Giachetti, C. Daffara, C. Reghelin, E. Gobbetti, R. Pintus, Light calibration and quality assessment methods for reflectance transformation imaging applied to artworks' analysis, in: SPIE Optical Metrology, International Society for Optics and Photonics, 2015, pp. 95270B95270B.

[40] J. Sun, X. Chen, Z. Gong, Z. Liu, Y. Zhao, Accurate camera calibration with distortion models using sphere images, Optics \& Laser Technology 65 (2015) 83-87.

[41] A. W. Fitzgibbon, R. B. Fisher, A buyer's guide to conic fitting (1995) 513-522. 
[42] M. Zhang, M. S. Drew, Robust luminance and chromaticity for matte regression in polynomial texture mapping, in: European Conference on Computer Vision, Springer, 2012, pp. 360-369.

[43] M. S. Drew, Y. Hel-Or, T. Malzbender, N. Hajari, Robust estimation of surface properties and interpolation of shadow/specularity components, Image and Vision Computing 30 (2012) 317-331.

[44] G. Sirat, D. Psaltis, Conoscopic holography, Optics letters 10 (1985) 4-6. 